\title{
SIMULATION OF ACOUSTIC EMISSION IN PLANAR CARBON FIBER REINFORCED PLASTIC SPECIMENS
}

\author{
M. G. R. SAUSE and S. HORN \\ University of Augsburg, Institute for Physics, Experimentalphysik II, D-86135 Augsburg
}

\begin{abstract}
The simulation of acoustic emission waveforms resulting from failure during mechanical loading of carbon fiber reinforced plastic structures is investigated using a finite element simulation approach. For this investigation we focus on the dominant failure mechanisms in fiber reinforced structures consisting of matrix cracking, fiber breakage and fiber-matrix interface failure. To simulate the failure process accurately, we present a new acoustic emission source model that is based on the microscopic source geometry and micromechanical properties of fiber and resin. We demonstrate that based on this microscopic source model these failure mechanisms result in excitation of macroscopic plate waves. The propagation of these plate waves is described using a macroscopic three-dimensional model geometry which includes contributions of reflections from the specimen boundaries. We further present a model of the acoustic emission sensors used in experiments to simulate the influence of aperture effects. To enhance the understanding of correlation between macroscopically detectable acoustic emission signals and microscopic failure mechanisms we simulate the response to different source excitation times, crack surface displacements and displacement directions. The results obtained show good agreement with fundamental assumptions about the crack process reported by various other authors. The simulated acoustic emission signals obtained are compared to experimentally measured waveforms during four-point bending experiments of carbon fiber reinforced plastic structures. The simulated signals of fiber-breakage, matrix-cracking and fiber-matrix interface failure show systematic agreement with the respective experimental signals.
\end{abstract}

\section{Introduction}

During mechanical loading of carbon fiber reinforced plastics (CFRP) various damage mechanisms occur. The accumulated damages finally result in failure of the composite. Typical damage mechanisms are matrix cracking, fiber-matrix debonding, fiber pull-out, fiber bridging, inter-ply failure and fiber breakage (see fig. 1). Depending on the CFRP application and the type of loading the significance of the various damage mechanisms for the composites integrity can change. In order to understand the respective contribution of these damage mechanisms to the ultimate failure of the composite it is necessary to record their evolution as a function of loading. Consequently, a nondestructive monitoring method of these damage mechanisms is necessary to understand the evolution of the failure process. 

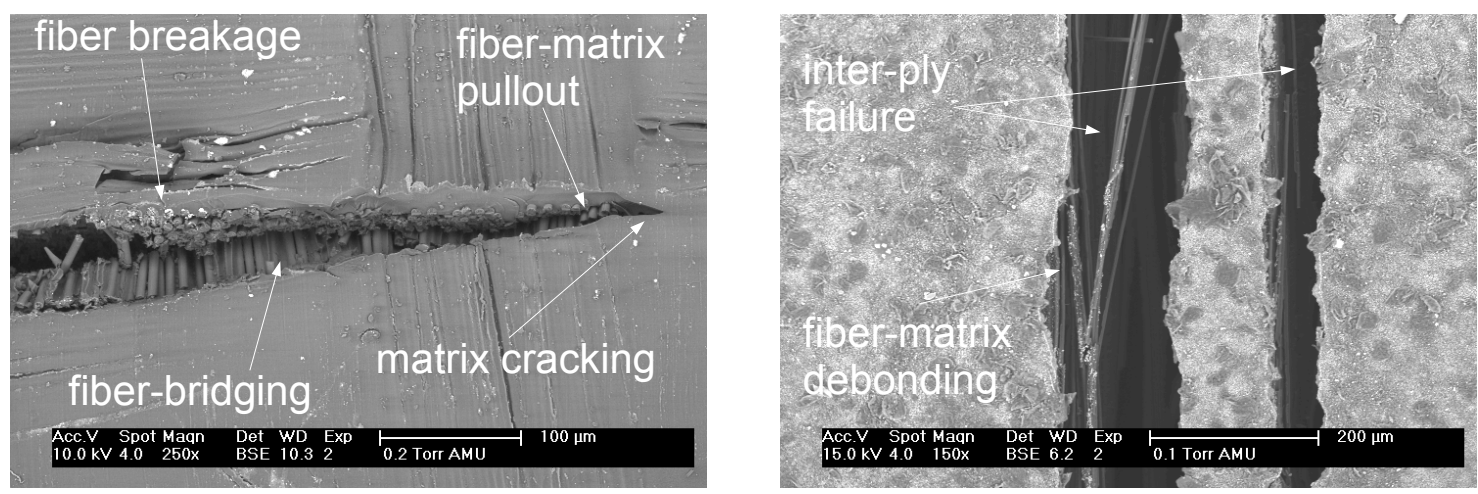

Fig. 1: Scanning electron microscopy images of typical damage mechanisms observed in carbon fiber reinforced plastics in top view (left) and side view (right).

A powerful tool to monitor micro-deformation processes is acoustic emission analysis. During formation of a crack surface a small density wave is released into the surrounding medium, which can be detected in the ultrasonic range by suitable piezoelectric sensors. Due to the rapid movement of the crack surface during formation, the excited elastic wave shows a broad frequency distribution.

In the past various authors have reported a correlation between certain damage mechanisms in fiber reinforced materials and the measured frequency composition of the associated acoustic emission signals. Several studies proposed to distinguish between fiber breakage and matrix cracking based on significant contributions at high frequencies (fiber breakage) or low frequencies (matrix cracking) [1, 2, 3, 4, 5]. Usually these observations use a combination of microscopy and localization of acoustic emission sources or use model composites in which one type of failure mechanism preferably occurs.

Alternatively, acoustic emission signals were calculated either by analytical approach or by finite element simulations. Following the early suggestions of Ono and Ohtsu acoustic emission sources can be modeled using buried dipole sources [6, 7]. Analytical calculations of the wave propagation itself were performed using Green Functions in infinite bodies, infinite half-spaces or infinite plates. Several authors extended this approach overcoming crucial limitations for CFRPs by introducing anisotropy [8] and a dissipative medium for wave propagation [9]. Another type of analytical approach to wave propagation was introduced using the integral formulation of generalized ray theory [10]. All these approaches contributed to the understanding of the nature of wave generation and the fundamental correlations between the excited waveforms and the size, shape and temporal evolution of the underlying acoustic emission source.

A connection between analytical solutions for acoustic emission in fiber reinforced structures and experiments was achieved by Giordano et al., who compared acoustic emission signals from a single fiber fragmentation test to theoretical solutions for wave propagation in an infinite Maxwell medium [9]. Here good agreement between the calculated and measured frequency spectra was found. This confirmed fundamental assumptions about acoustic emission signals propagating in a viscoelastic medium with density $\rho$ and bulk modulus $E$ and the correlation to frequency spectra of waveforms detected at the specimen surface.

All these analytical solutions assume an infinite extension of at least one of the propagation medium's axis. To overcome these limitations, an approach to modeling of acoustic emission signal propagation was published by Prosser et al. who 
successfully established a finite element simulation based on an anisotropic graphite/epoxy medium to include boundary reflections [11]. The acoustic emission source was modeled as buried dipole source with a force pulse as source mechanism. Recently Wilcox et al. presented an approach on forward-modeling of the evolution of acoustic emission from excitation to detection [32, 33]. Based upon the concept of transfer functions the approach emphasizes the separability of contributions from the source mechanism, the propagation and the detection. In particular the presented methodology is able to combine the obtained transferfunctions from analytical solutions, finite element simulations and experimental results. The so obtained forward predictions in composite structures show very good agreement to experimental data [33].

In fiber reinforced structures of plate-like geometry the movement of the microscopic crack surface excites plate waves that propagate through the macroscopic structure. In finite element simulations the propagation of ultrasonic waveforms is modeled based on time dependent solutions of differential equations of the equilibrium states. The excitation of ultrasonic waveforms is usually achieved by a driving force acting on one of the faces of the plate-like geometry [12]. This model assumption is a particularly good approximation to ultrasonic waveform generators, like piezoelectric elements. Nieuwenhuis et al. established a finite element model that coupled the displacement of a lead zirconate titanate (PZT) element mounted on a plate with an out-of-plane surface displacement [13]. It was demonstrated that the waveforms excited by the modeled piezoelectric element are nearly equivalent to the waveforms generated by a driving force applied directly to the same area. This is in agreement with Lamb-wave measurements in experimental specimens [14].

In contrast to small sized specimens, the waveform propagation in larger viscoelastic specimens is influenced by attenuation and dispersion of waveforms which, consequently, should be included in the model. To this end, Castaings et al. established an alternative approach to simulate ultrasonic waveform propagation in viscoelastic media. Instead of the computationally burdensome approach in the time domain, they solved the time dependent equations of the equilibrium states in the frequency domain for a limited number of frequencies. The temporal propagation was deduced using Fourier-Transformations [12]. To include the effects of attenuation they introduced complex elastic coefficients, which were measured utilizing a specifically developed immersion method [15]. The obtained results show good agreement with measurements on model specimens and confirm the importance of these effects for waveform propagation in viscoelastic media [12].

Finite element simulations by Hamstad et al. already have shown that different source radiation directions can stimulate different ratios of symmetric and antisymmetric plate wave modes in isotropic materials [16]. This in turn can lead to different frequency compositions of the detected acoustic emission signals, as the propagation of the zero-order symmetric $\left(S_{0}\right)$ mode occurs at higher frequency than the zero-order antisymmetric $\left(A_{0}\right)$ mode [17]. Due to the microscopically anisotropic nature of fiber reinforced composites, depending on the damage mechanism strong differences in the source radiation direction are expected.

\section{Description of simulation and source model}


The generation of ultrasonic waveforms caused by micro-mechanical deformations is commonly called acoustic emission. In CFRPs the micro-deformation process of interest is the formation of cracks within fiber, matrix or at the interface between them. According to the principles of fracture mechanics the system moves from one equilibrium state (before crack progress) to another equilibrium state (after crack propagation). The energy release during crack propagation is consumed by plastic deformation, heat generation and the generation of elastic waves propagating into the solid.

Based on the principle of virtual work $W$ the Comsol program solves the dynamic equations of equilibrium, expressed in global or local stress and strain vector components $\vec{\sigma}$ and $\vec{\varepsilon}$ for an external stimulation [19]. The acoustic emission waveforms are then deduced from the respective $\vec{\varepsilon}$-components at the model geometry's surface in the area of detection.

The principle of virtual work states that the variation of $W$ induced by forces $F_{i}$ and virtual displacements $\delta u_{i}$ in an equilibrium state equals zero:

$$
\delta W=\sum_{i} F_{i} \cdot \delta u_{i}=0
$$

Generally, the external applied virtual work equals the internal virtual work and in the case of a deformable body with volume $V$ and surface $S$, results in a deformation state with new internal stress and strain components.

$$
\int_{S} \delta \vec{u}^{t} \cdot \vec{F}_{S} d S+\int_{V} \delta \vec{u}^{t} \cdot \vec{F}_{V} d V-\int_{V} \delta \vec{\varepsilon}^{t} \cdot \vec{\sigma} d V=0
$$

The external applied forces $\vec{F}_{S}$ and $\vec{F}_{V}$ act on the surface and volume of the body, respectively. The constraint forces within the material are expressed by consistent internal stress $\vec{\sigma}$ and strain $\vec{\varepsilon}$ components, with the superscript $t$ indicating the transposed vectors.

Assuming that the only source of stress $\vec{\sigma}$ is due to strain $\vec{\varepsilon}$ resulting from crack progress, for linear elastic media Hooks law is valid.

$$
\vec{\sigma}=\vec{D} \cdot \vec{\varepsilon}
$$

In the general case for anisotropic media the elasticity tensor $\vec{D}$ is a $6 \times 6$ matrix with 12 independent components. The stress vector $\vec{\sigma}$ has six independent components composed of normal stresses $\sigma$ and shear stresses $\tau$. The strain vector $\vec{\varepsilon}$ also has six independent components consisting of normal components $\varepsilon$ and shear strain components $\gamma$.

$$
\left(\begin{array}{l}
\sigma_{x} \\
\sigma_{y} \\
\sigma_{z} \\
\tau_{x y} \\
\tau_{y z} \\
\tau_{x z}
\end{array}\right)=\left(\begin{array}{cccccc}
D_{11} & D_{12} & D_{13} & 0 & 0 & 0 \\
D_{21} & D_{22} & D_{23} & 0 & 0 & 0 \\
D_{31} & D_{32} & D_{33} & 0 & 0 & 0 \\
0 & 0 & 0 & D_{44} & 0 & 0 \\
0 & 0 & 0 & 0 & D_{55} & 0 \\
0 & 0 & 0 & 0 & 0 & D_{66}
\end{array}\right) \cdot\left(\begin{array}{c}
\varepsilon_{x} \\
\varepsilon_{y} \\
\varepsilon_{z} \\
\gamma_{x y} \\
\gamma_{y z} \\
\gamma_{x z}
\end{array}\right)
$$


In the case of isotropic media the elasticity tensor is completely described by Young's modulus $E$ and the Poisson number $v$.

To extend the principle of virtual work for dynamic systems, mass accelerations are introduced. This yields the formulation of the d'Alembert principle which states that a state of dynamic equilibrium exists if the virtual work for arbitrary virtual displacements vanishes. Taking this into account and introducing the material density $\rho$ equation (2) becomes:

$$
\int_{S} \delta \vec{u}^{t} \cdot \vec{F}_{S} d S+\int_{V} \delta \vec{u}^{t} \cdot \vec{F}_{V} d V-\int_{V} \delta \vec{\varepsilon}^{t} \cdot \vec{\sigma} d V-\int_{V} \rho \frac{\partial^{2} \vec{u}}{\partial t^{2}} \cdot \delta \vec{u} d V=0
$$

This defines the basic differential equation solved within the Comsol environment for every finite element.

To compare simulated with experimentally obtained acoustic emission waveforms we used a bending specimen geometry adapted to the requirements of DIN-EN-ISO 14125 (right panel of figure 2). The modeling of the complete specimen was realized using the Structural Mechanics Module of Comsol Multiphysics [19]. The used model geometry shown on the left of figure 2 contains one quarter of the volume of the experimental specimen. In order to reduce the computation time, two planes of symmetry (yz-plane and $x z$-plane) were used. In order to take into account both acoustic waveform generation and waveform propagation a multi-scale approach was chosen.

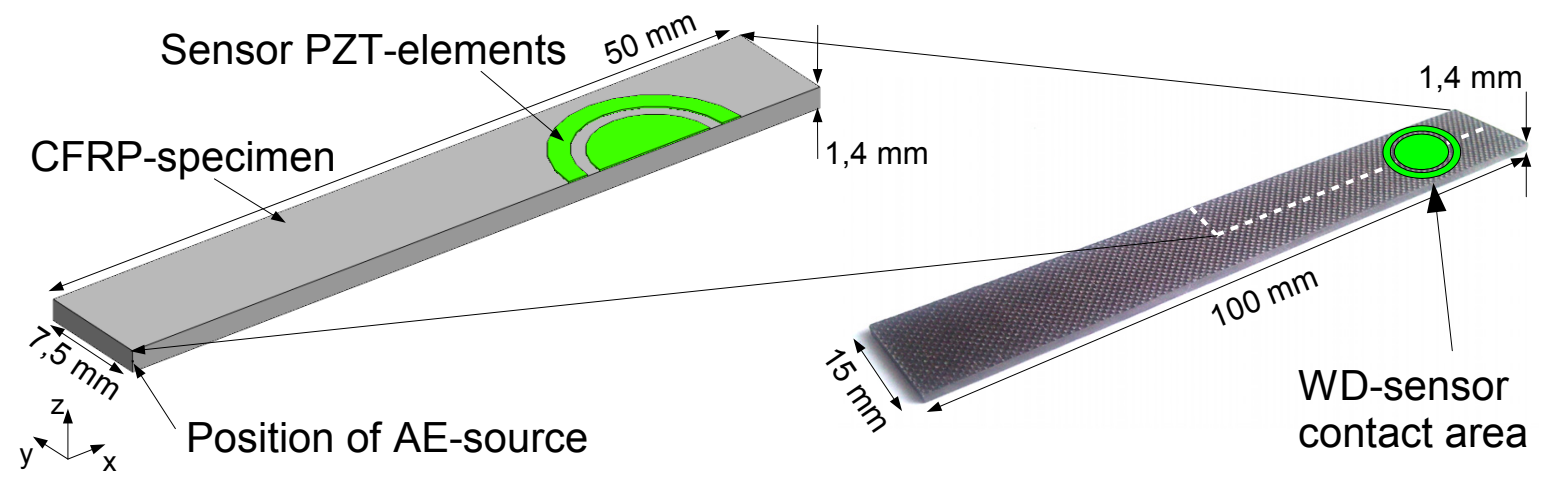

Fig. 2: Model geometry (left) and experimentally used specimen (right). The geometry is adapted to DIN-EN-ISO 14125 bending specimens.

The maximum mesh element scale was $0.5 \mathrm{~mm}$ on the macroscopic scale, with a gradually decrease in scale when approaching the acoustic emission source. The sufficiency of this mesh resolution was confirmed by the fact that doubling the mesh resolution in the vicinity of the model source and increasing the resolution on the macroscopic scale to $0.3 \mathrm{~mm}$ resulted in no changes of the model calculations.

The elastic coefficients of the materials used in the simulation process are summarized in table 1. Due to a lack of experimentally available values for the CFRP specimens investigated, we applied literature values from Prosser et al. to model the specimens elastic properties [11]. Since the elastic coefficients are in reasonable agreement with the basic specimen properties of the measured values $D_{11}$ (152 GPa) and $\mathrm{D}_{22}(7,4 \mathrm{GPa})$ from tensile testing in $0^{\circ}$ and $90^{\circ}$ fiber orientation the application of 
literature values appears justified. To model the elastic properties of the pure resin and the carbon fibers we used values from the manufacturer datasheets.

Since the exact material composition of the piezoelectric sensor material used in our investigation is not available the anisotropic elastic coefficients were taken from [13] for a Motorala 3203HD PZT.

The chosen range of excitation times investigated is between 50 ns and $3 \mu$ s. These values are based on the reported excitation times by Hatano et al. and Giordano et al. who both used $1 \mu \mathrm{s}$ as typical source rise time [24, 9]. In order to resolve the smallest chosen excitation time of $\mathrm{T}_{\mathrm{e}}=50 \mathrm{~ns}$ in the time domain, the initial time step of the model was set to $10 \mathrm{~ns}$. To decrease the amount of data the time steps were gradually increased after excitation to reach a final temporal interval of $\Delta t=0.1 \mu \mathrm{s}$. This resolution corresponds to an experimental sampling rate of $10 \mathrm{MHz}$ and is sufficient to resolve the observed signals frequency content in the range up to a maximal frequency of $5 \mathrm{MHz}$.

\begin{tabular}{|l|l|l|l|l|}
\hline Physical Property & $\begin{array}{l}\text { Unidirectional } \\
\text { CFRP }\end{array}$ & Resin Hexply 913 & $\begin{array}{l}\text { Carbon Fiber } \\
\text { T800 }\end{array}$ & $\begin{array}{l}\text { PZT Motorola } \\
\text { 3203HD }\end{array}$ \\
\hline Density r [kg/m ${ }^{3}$ ] & 1550 & 1230 & 1810 & 7500 \\
\hline Poisson-Ratio & - & 0,35 & 0,20 & - \\
\hline Elasticity & $D_{11}=147,1$ & $\mathrm{E}=3,39$ & $\mathrm{E}=294$ & $\mathrm{D}_{11}=142,0$ \\
Constants [GPa] & $\mathrm{D}_{12}=4,11$ & & & $\mathrm{D}_{12}=91,9$ \\
& $\mathrm{D}_{13}=4,11$ & & & $\mathrm{D}_{13}=102,0$ \\
& $\mathrm{D}_{22}=10,59$ & & & $\mathrm{D}_{22}=142,0$ \\
& $\mathrm{D}_{23}=3,09$ & & $\mathrm{D}_{23}=102,0$ \\
& $\mathrm{D}_{33}=10,59$ & & & $\mathrm{D}_{33}=141,0$ \\
& $\mathrm{D}_{44}=3,75$ & & & $\mathrm{D}_{44}=25,5$ \\
& $\mathrm{D}_{55}=5,97$ & & & $\mathrm{D}_{55}=25,5$ \\
& $\mathrm{D}_{66}=5,97$ & & & $\mathrm{D}_{66}=25,1$ \\
\hline Sources: & {$[11]$} & [Datasheet] & [Datasheet] & {$[13]$} \\
\hline
\end{tabular}

Table 1: Summary of material properties used for the simulation of acoustic emission waveforms in this investigation.

Although the currently used source representations are quite valuable to model the excitation of acoustic emission signals $[6,7,8,9,11,34]$, they do not focus in detail on the microscopic elastic properties of the cracking medium and the exact geometry of the crack with respect to the surrounding medium. There are two ways to obtain a more realistic model for acoustic emission excitation. On the one hand, the source can be described by the force distribution around the crack tip, on the other hand realistic parameters describing crack propagation are the time-dependence and displacement amplitude of the newly formed crack surface. The elastic wave excitation itself results from microscopic oscillations of the cracks surface [18].

To describe the acoustic emission from such crack progress, we introduce a new acoustic emission source model based on a short temporal displacement of the crack surface of finite extension as shown in figure 3. To this end the input parameters of our model source are solely the magnitude of displacement, which is correlated to the strain energy before fracture and a characteristic time for the crack-formation. The presented source geometry includes spatial dimensions of the crack surface and effects of orientation between fiber axis and the crack surface movement direction. This consists of a rectangular model-fiber with cross-section $10 \times 10 \mu \mathrm{m}^{2}$ and $30 \mu \mathrm{m}$ length is embedded in a resin cube of $100 \mu \mathrm{m}$ edge length. To simulate pure matrix 
cracking, the model-fibers elastic properties were replaced by the elastic properties of the resin.

To simulate the displacement of the crack surface, a cross-shaped volume with dimensions given in figure 4 was cut out of the resin cube. This enables quasiindependent movement of the "cracks" surfaces in $\mathrm{x}, \mathrm{y}$, or $\mathrm{z}$ direction. The advantage of this cross-shape is the possibility to simulate all possible combinations of radiation directions without remeshing the model, which could in turn influence the comparability of the calculation result. In our investigation, the acoustic emission source was always centered with respect to the $x$ - and $y$-direction of the specimen volume, while its vertical position within the plate was varied to investigate its influence on the excitation of plate-waves. To excite an acoustic emission waveform a displacement $\mathrm{d}_{(\mathrm{xyz})}$ of the model crack surface which increases linear in time for a time interval $T_{e,(x y z)}$ was introduced. The $(x y z)$ subset indicates the respective displacement direction.

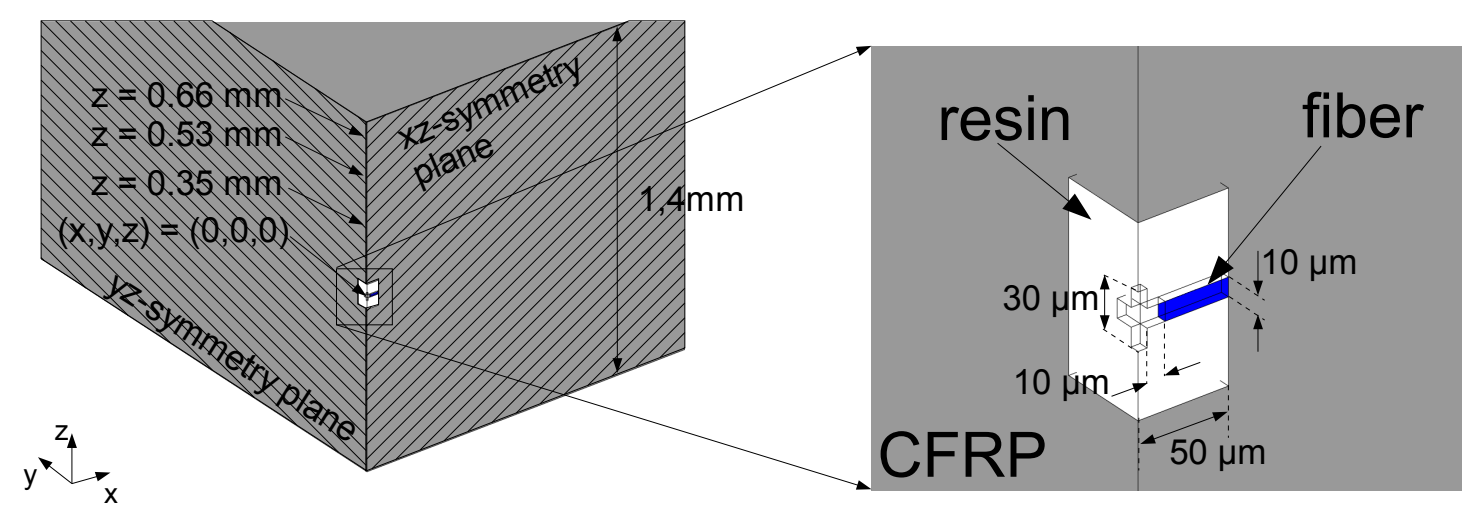

Fig. 3: Acoustic emission source model used in this investigation. The yz-plane and $x z$-plane were chosen as symmetry plane.

As a response to such a short temporal displacement the crack source starts to oscillate and is damped out as the energy is propagating into the solids volume as shown in figure 4 . We want to point out that the vibrational response of the crack surface shown in figure 4 is attributed solely to the models elastic properties and not to a driving force like commonly used in other publications [12, 14, 13]. Based on identical excitation times $T_{e, x}=50 \mathrm{~ns}$ and displacements $d_{x}=100 \mathrm{~nm}$ the different elastic properties of fiber and resin result in characteristic source spectra as shown in figure 5 . For the case of a simulated fiber breakage the bandwidth of the frequency spectra is broader than that for matrix cracking, which is a result of the drastically different elastic properties as expected from the theory of Giordano et al. [9]. In addition the amplitude of the elastic wave of the simulated fiber breakage is significantly higher. 


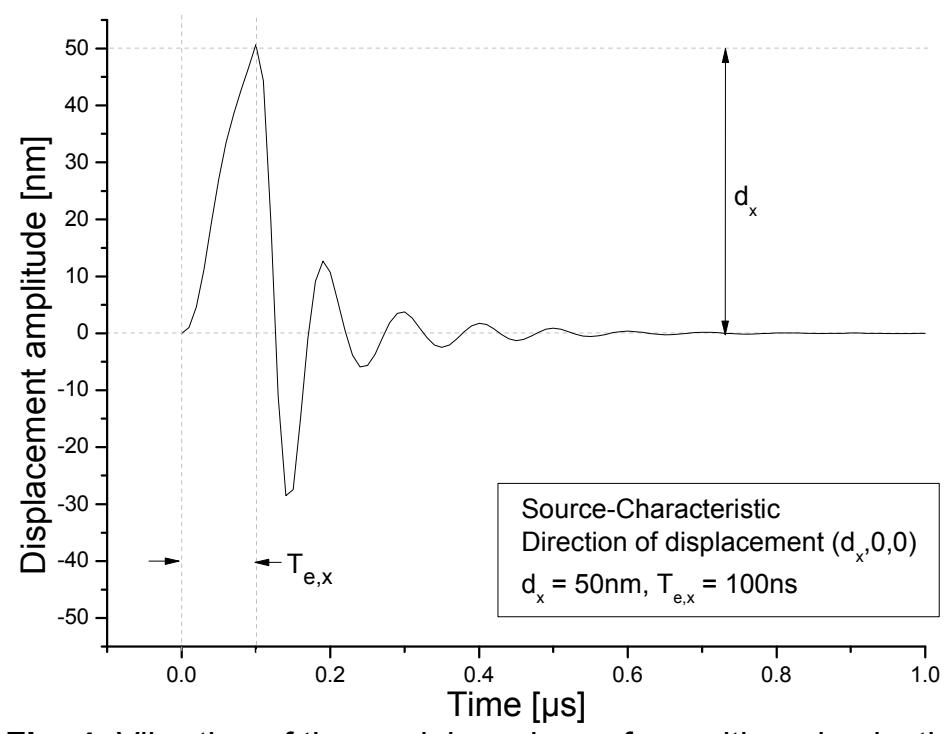

Fig. 4: Vibration of the model cracks surface with resin elastic properties for an excitation with $T_{e, x}=$ $100 \mathrm{~ns}$ and displacement $d_{x}=50 \mathrm{~nm}$. The vibrational response is attributed solely to the elastic properties of the model and was not introduced by an oscillating driving force.

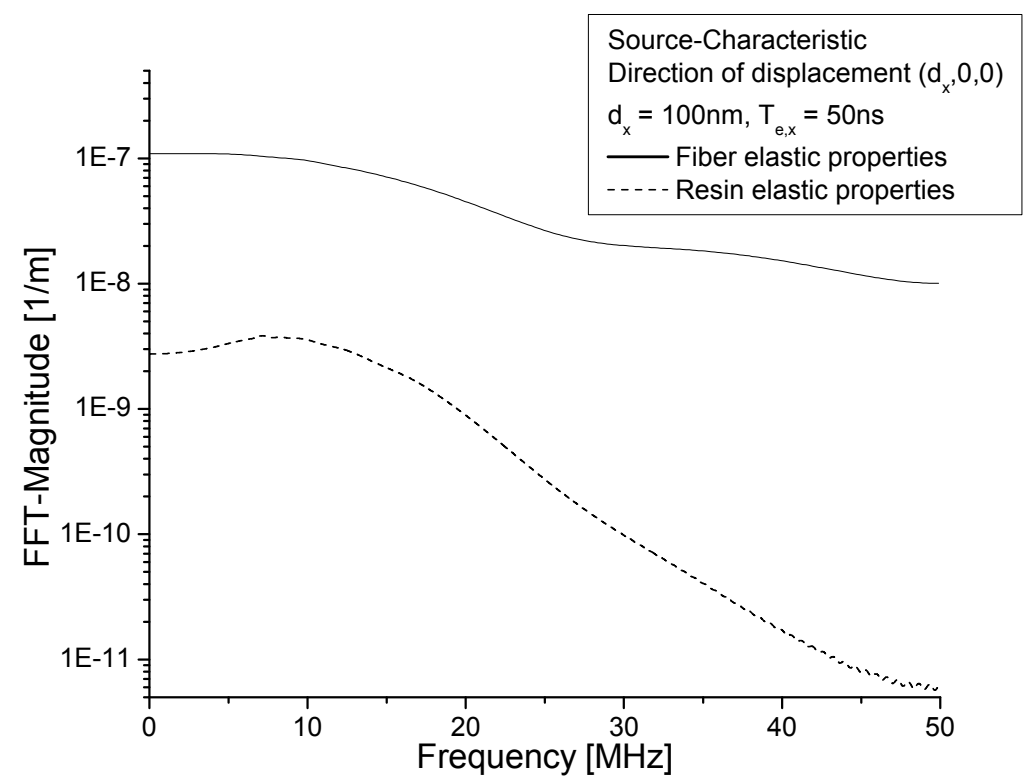

Fig. 5: Source spectra obtained from the crack surface vibration in $x$-direction for an excitation with $T_{e, x}=50 \mathrm{~ns}$ and displacement $d_{x}=100 \mathrm{~nm}$ for fiber and resin elastic properties.

We want to point out, that the broad frequency spectra of the source mechanism suffer from significant bandwidth reduction during propagation and especially during detection with a bandwidth limited sensor.

The displacement fields excited by the crack surface displacements used to simulate the different failure mechanisms investigated are summarized in figure 6 . In the following the fibers are oriented along the x-axis in a planar composite with plate length in $\mathrm{x}$ - and width in $\mathrm{y}$-direction, which is the usual structure of a fiber reinforced composite plate. The $z$-axis direction is chosen normal to the plate surface. 


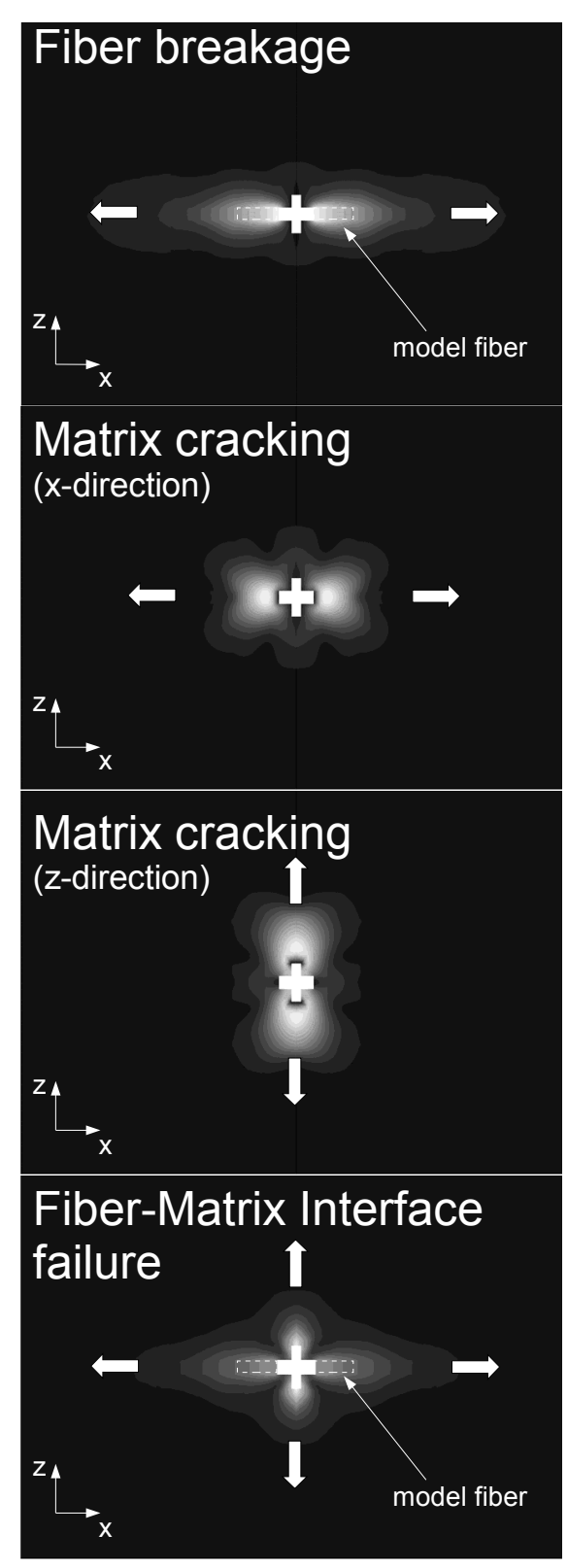

Fig. 6: Magnitude of the displacement vector in xz-cross-section plane mirrored around yz-symmetry plane for the different simulated failure mechanisms obtained from the simulation at $t=50 \mathrm{~ns}$. The arrows indicate the direction of the chosen initial displacement $d_{(x y z)}=100 \mathrm{~nm}$, while the excitation time for all processes was kept constant at $\mathrm{T}_{\mathrm{e}}=50 \mathrm{~ns}$.

For fiber breakage the major displacement is directed along the fiber axis, i.e. the dominant source radiation direction is in the xy-plane (in-plane). The magnitude of the displacement vector $\vec{r}=\sqrt{x^{2}+y^{2}+z^{2}}$ shown in figure 6 yields the typical dipolecharacteristic commonly associated with an in-plane tensile crack $[6,7,8,10,16,17$, 35].

For matrix cracking the crack surface normal can be directed along any axis. This was modeled by short temporal displacement along the $x-, y-$ or $z$-axis. The displacement vector magnitudes show source radiation patterns which can also be approximated by a dipole characteristic. For displacement in $x$ - or $y$-direction (equivalent) this is an in-plane dipole, for displacement in z-direction this is an out-ofplane dipole, respectively. While the in-plane dipoles represent matrix cracking, the out-of-plane dipoles should be understood to represent inter-ply delamination in matrix rich areas. In contrast to the displacement field of the simulated fiber breakage 
the displacement field of the matrix cracks appears to be distorted from a pure dipole. This is a result of the different elastic properties of the cracking medium, i.e. carbon fiber and resin, and the anisotropic elastic properties of the surrounding solid. During crack progress all interface processes which involve fiber and matrix (e.g. debonding) should contribute to in-plane and out-of-plane radiations. In this case, the crack formation at the interface is simulated as a z-axis displacement perpendicular to the crack surface. In addition, due to the loss of contact between fiber and matrix a relaxation of the fiber occurs. Consequently, an additional $x$-axis displacement component (along the fiber axis) is simulated. The obtained field of the displacement vector magnitude is best approximated by a quadrupole characteristics, which is a result of these two different displacements and the anisotropic properties of the sources surrounding.

We want to point out, that $\vec{\nabla} \vec{r}=\left(\frac{\partial \vec{r}}{\partial x}, \frac{\partial \vec{r}}{\partial y}, \frac{\partial \vec{r}}{\partial z}, \frac{\partial \vec{r}}{\partial t}\right)$ is continuous when crossing the border between the embedded source model and the macroscopic CFRP specimen. This elucidates that this discontinuous change of elastic properties introduced in our multi-scale model does not drastically influence the signal excitation and propagation.

The propagation of the acoustic emission signal is dominated by the mean elastic properties of the propagation medium. To model the elastic properties of unidirectional CFRP-specimens we used real anisotropic elastic coefficients. This is sufficient to simulate guided waves in viscoelastic media if attenuation effects arising from dispersive Lamb-wave propagation are neglected during propagation. As discussed by Prosser et al. the dominating part for attenuation in the near-field range within the distance $x<4.34 / \alpha$ is geometric spreading [17]. In our specimens the propagation distance between source and area of detection is about $40 \mathrm{~mm}$, while the attenuation $\alpha$ is $100+/-10 \mathrm{~dB} / \mathrm{m}$. As a consequence the attenuation is dominated by geometric spreading, which is intrinsically included within our model.

The temporal development of the magnitude of the displacement vector in figure 7 demonstrates the near-field propagation of the acoustic emission waveform based on a simulation with excitation time $T_{e, x}=0.3 \mu$ s and $x$-displacement of $d_{x}=100 \mathrm{~nm}$. In this case, the dominant contribution to the displacement vector results from the excitation in x-direction. This direction of excitation stimulates high intensity $\mathrm{S}_{0}$ and a low intensity $A_{0}$ plate wave mode. This can be seen in more detail in figure 8 in the far-field propagation of the acoustic emission waveform. As expected, the $S_{0}$ mode travels at higher speed and reaches the sensor position before the $A_{0}$ plate wave mode. In contrast, for excitations in z-direction a strong excitation of the $A_{0}$ mode was observed. A similar observation was reported by Hamstad et al. for the case of isotropic materials [16]. 
(a)

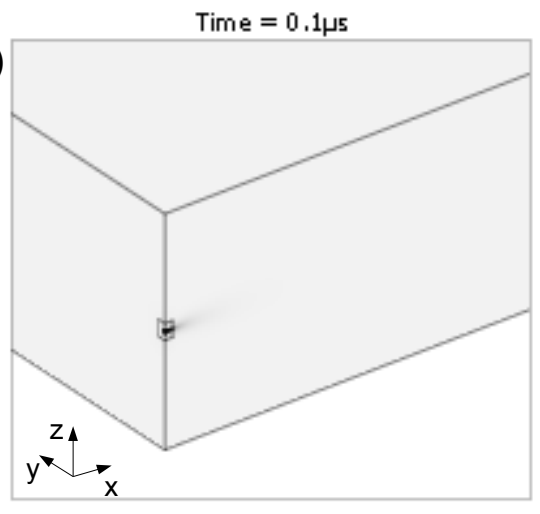

Max: $3.34 \mathrm{e}-8$

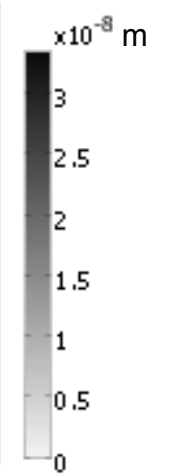

(c)

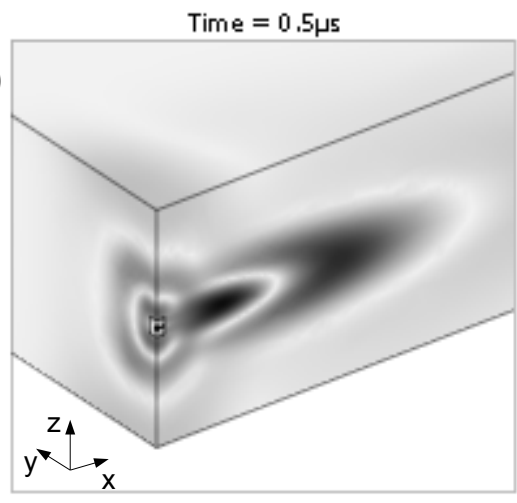

Max: $3.73 \mathrm{e}-10$

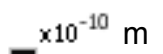

3.5

1.5

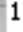

0.5 (b)

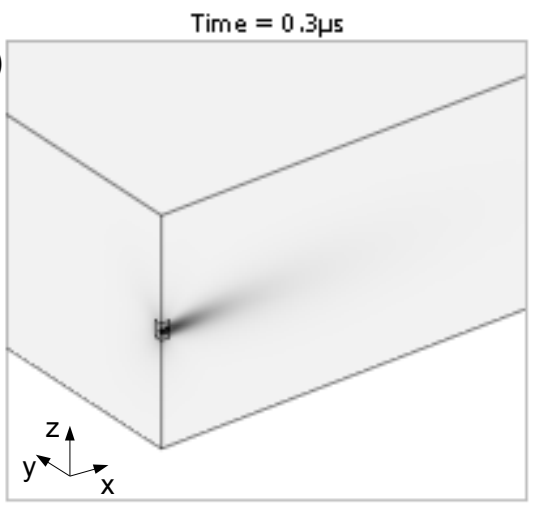

Max: $7,45 e-9$

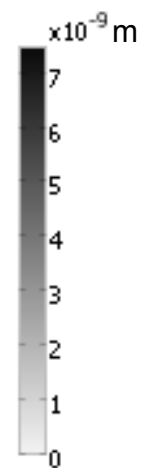

(d)

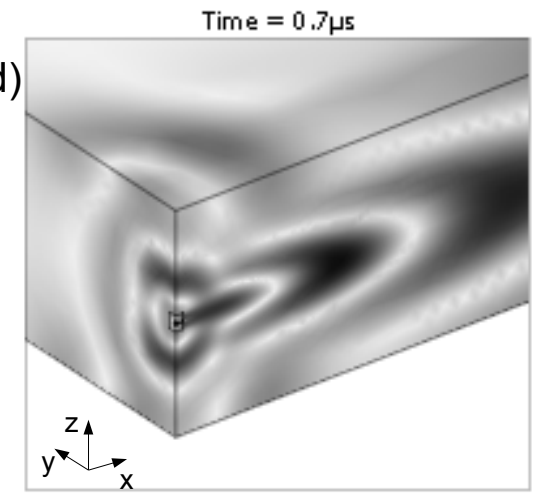

Max: $5.90 \mathrm{e}-11$

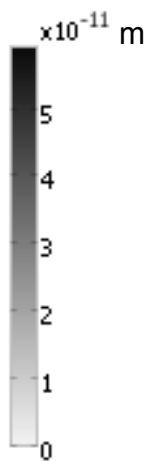

Fig. 7: Temporal development of the magnitude of the displacement vector in the near-field region of the acoustic emission source for a simulation of fiber-breakage. Times shown are $0.1 \mu \mathrm{s}(\mathrm{a}), 0.3 \mu \mathrm{s}$ (b), $0.5 \mu \mathrm{s}$ (c) and $0.7 \mu \mathrm{s}$ (d) after excitation (excitation time $T_{e, x}=0.3 \mu \mathrm{s}, x$-displacement $d_{x}=100 \mathrm{~nm}$ ). 

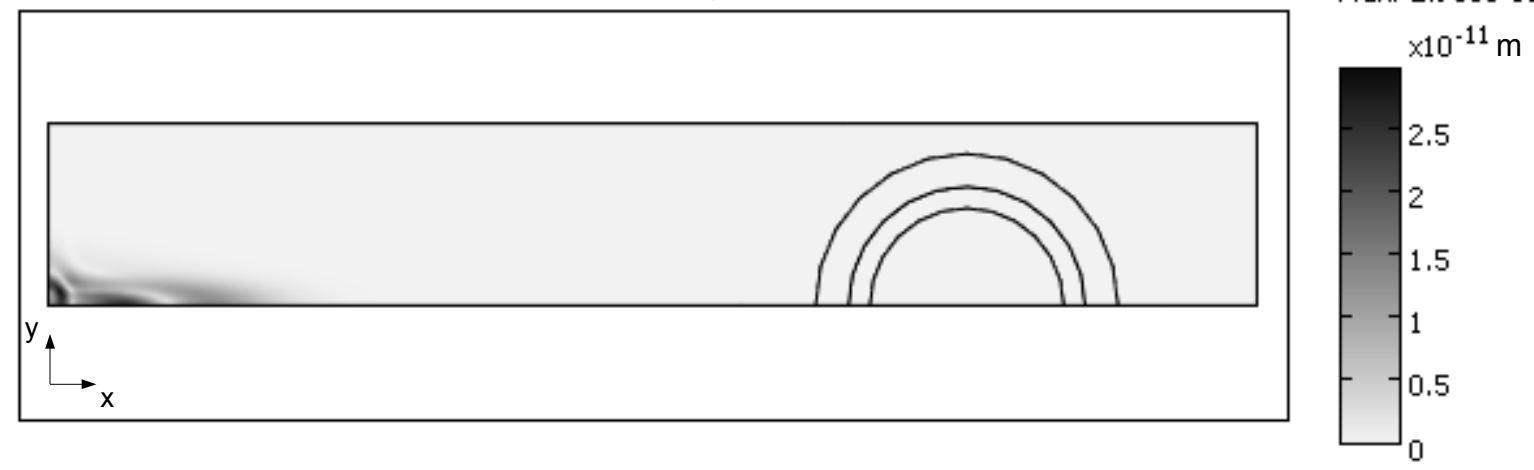

(b)

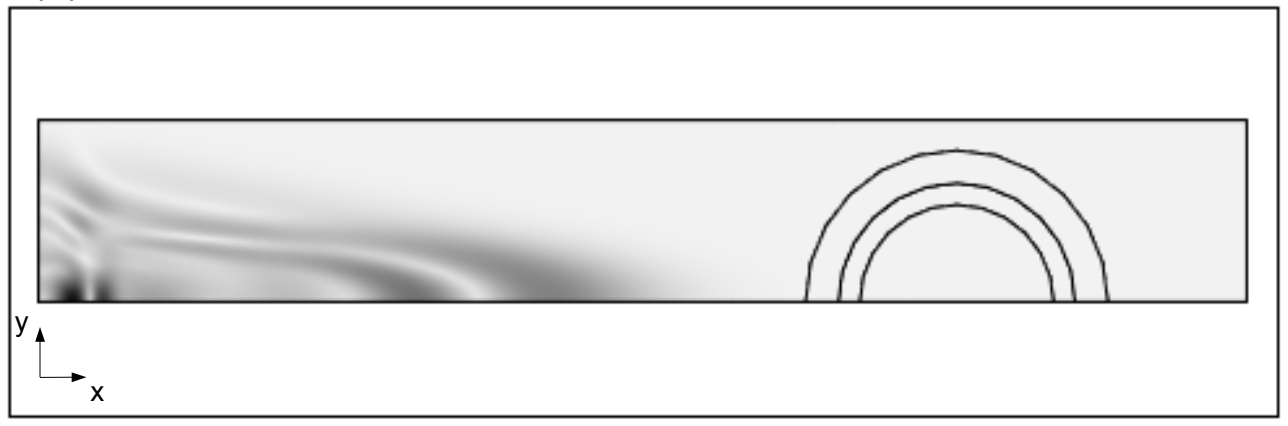

Max: $6.408 \mathrm{e}-12$
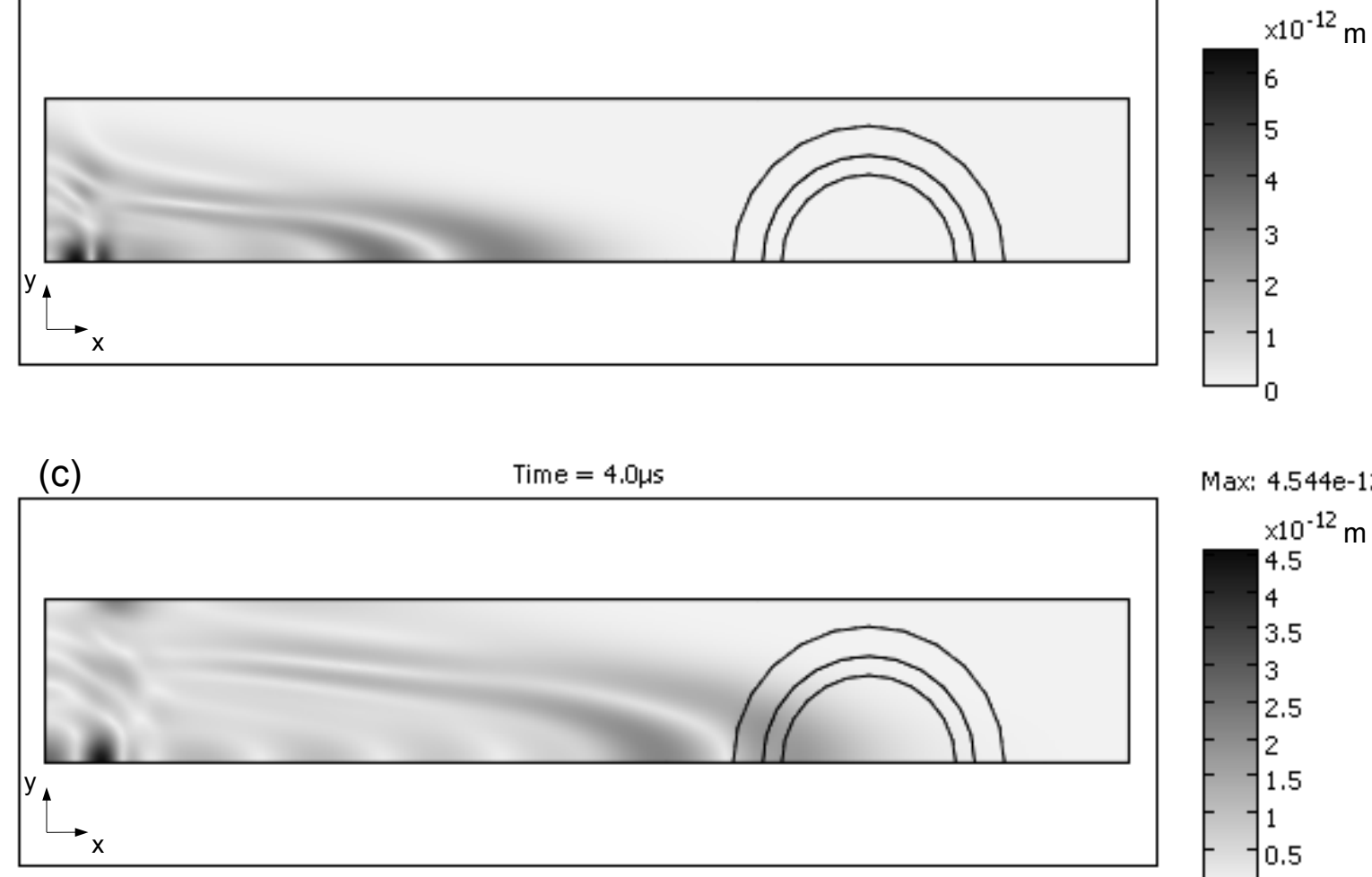

$\operatorname{Max}: 4.544 \mathrm{e}-12$

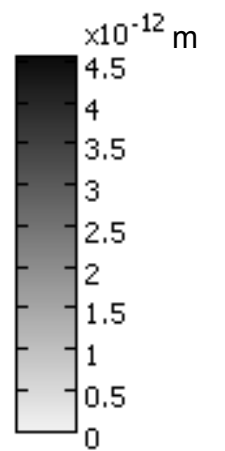

Min: 0

Fig. 8: Temporal development of the magnitude of the displacement vector in the far-field region of the acoustic emission source for a simulation of fiber-breakage. Times shown are $1.0 \mu \mathrm{s}$ (a), $2.5 \mu \mathrm{s}$ (b) and $4.0 \mu \mathrm{s}$ (c) after excitation (excitation time $\mathrm{T}_{\mathrm{e}, \mathrm{x}}=0.3 \mu \mathrm{s}$, $\mathrm{x}$-displacement $\mathrm{d}_{\mathrm{x}}=100 \mathrm{~nm}$ ).

The frequency and energy content of the measured signals depends on the sensor geometry. Commonly used acoustic emission sensors exhibit different piezoelectric element geometries. Several authors already demonstrated that the aperture effect of these sensor types on the detected signals is not negligible [20]. Moreover, Ono et al. have elucidated the fact that the sensor of type WD used in our investigation primarily responds to the surface velocity motion $v_{z}$ rather than to surface displacement [21]. In order to compare the result of the simulations to experimental data, the shape of the piezoelectric elements used within the type WD sensor as obtained by cross-sectional images was realized in the simulation (see figure 2). This approach takes into account the aperture effect of the experimentally used sensor, but is not meant to be a full-scale simulation of the detection process. To this end the complex geometry of the sensors interior and the piezoelectric conversion should be 
taken into account to yield signals, which are directly comparable to experimental data. Since the coupling between mechanical displacement of the piezoelectric element and the electrical charge displacement was not taken into account, no absolute values of signal voltages were calculated.

As mentioned above, the acoustic emission signal $\mathrm{S}(\mathrm{t})$ of the sensor used in this investigation is proportional to the surface velocity. To obtain the average surface velocity over the sensor area A defined by the two sensor elements (disc and ring) we integrate the $z$-component of the velocity $v_{z}$ of the surface displacement over $A$ and divide it by the respective contact area:

$$
S(t)=\frac{1}{A_{\text {disc }}} \int_{A_{\text {dise }}} v_{z}(t) d x d y+\frac{1}{A_{\text {ring }}} \int_{A_{\text {ring }}} v_{z}(t) d x d y
$$

In order to validate the sensor model we compare an experimental signal obtained from a pencil-lead fracture test to a simulated signal as shown in figure 9. The experimental signal was obtained from a pencil lead fracture at a distance of $60 \mathrm{~mm}$ away from the sensor applied on a $50 \mathrm{~mm} \times 16 \mathrm{~mm} \times 300 \mathrm{~mm}$ (width $\times$ height $\times$ length) steel plate. The simulated pencil lead fracture was realized as $3 \mathrm{~N}$ point force with a risetime of $300 \mathrm{~ns}$ according to [23] in a respective geometry. The signal obtained from the simulated average surface velocity $S(t)$ and the experimental signal show systematic agreement. For the purpose of discussion of the simulated signals in terms of significant frequency weight the obtained agreement is sufficient.

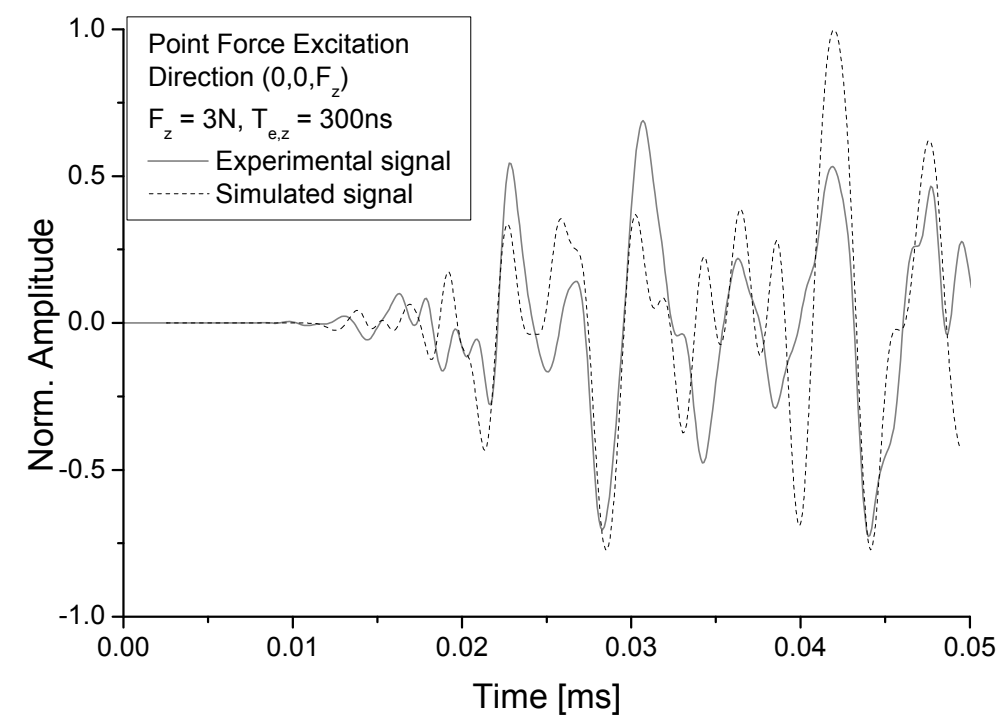

Fig. 9: Experimental signal obtained from a pencil-lead fracture on a steel plate compared to a simulated signal.

A comparison between the signals of the disc-element, the ring-element and the superposition of both clearly demonstrate the strong influence of the aperture effect of the chosen sensor geometry as shown in figure 10. The shape and the frequency content of the signals measured using a finite sensor element cannot be compared to a point displacement measured at the midpoint of the sensors position. 


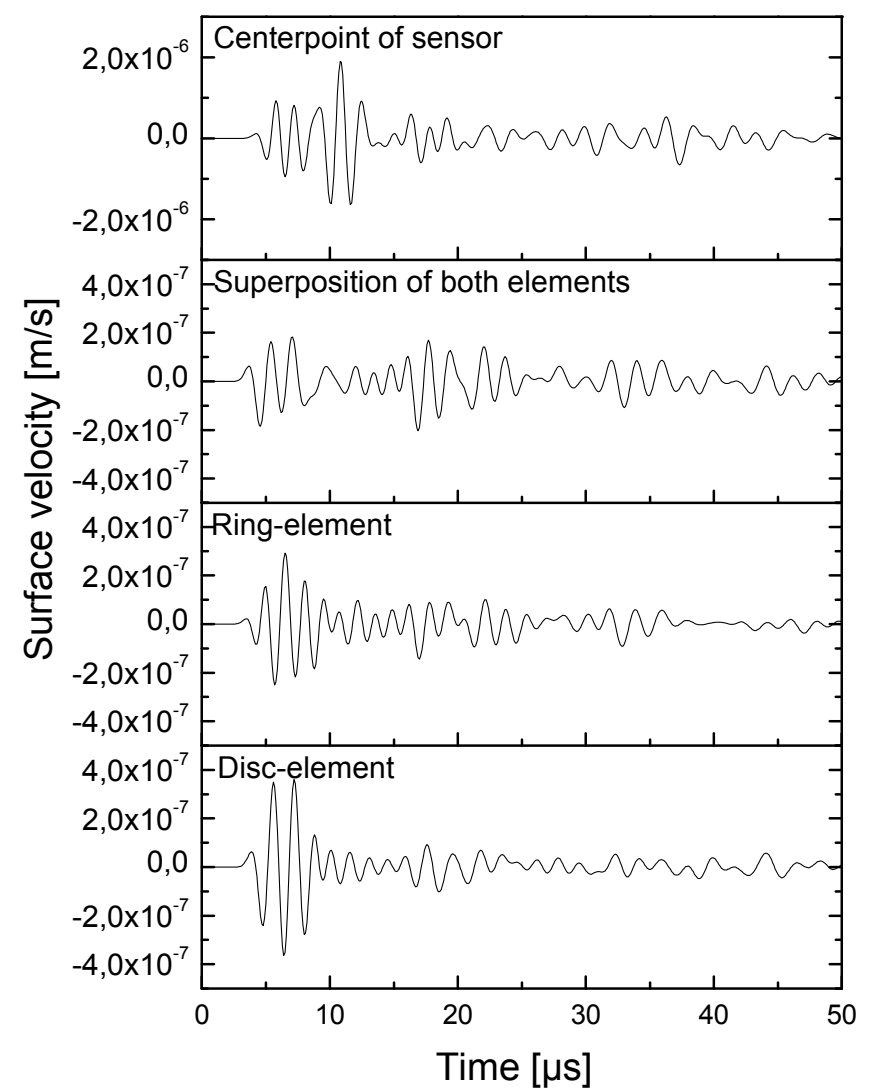

Fig. 10: Surface velocity response for different sensor geometry compositions for a simulation with excitation time $T_{e, x}=0.3 \mu$ s and $x$-displacement $d_{x}=100 \mathrm{~nm}$.

The simulated surface velocities of a point like area of the surface are between $10^{-}$ ${ }^{7} \mathrm{~m} / \mathrm{s}$ and $10^{-5} \mathrm{~m} / \mathrm{s}$, which agree well with the experimental values obtained by other authors during sensor calibration [24, 21]. For the investigation presented here the signal was calculated by integrating over the sensors contact area, i. e. by a superposition of both active piezoelectric elements. The combination of both elements can result in a double detection of a signal, since the acoustic emission signal is first detected at the ring-element and later (time-shifted) at the disc-element.

The calculated range of the simulated surface displacements at the sensor position due to the acoustic emission waveforms is in the order of $10^{-13} \mathrm{~m}$ to $10^{-10} \mathrm{~m}$. In comparison, pencil lead fracture (Hsu-Nielsen source) typically produces surface displacements of the order of $10^{-9} \mathrm{~m}[22,23]$. In our experiments pencil lead fracture results in intense energetic acoustic emission signals, which are much higher than those measured for microscopic crack formation investigated here. So in this case the simulated surface displacement range is very reasonable.

Figure 11 shows the interaction behavior between the sensor elements and the plate wave at the arrival time of the $\mathrm{S}_{0}$-mode. Since the displacement-field is disturbed by the presence of the piezoelectric elements, their influence on the waveform propagation and detection has to be taken into account. The importance of sensorspecimen interaction was already pointed out by Goujon et al., who demonstrated that the reception sensitivity of acoustic emission sensors does not only depend on the geometry, but also on the acoustic impedance ratio of the used sensor and specimen [20]. 
(a)

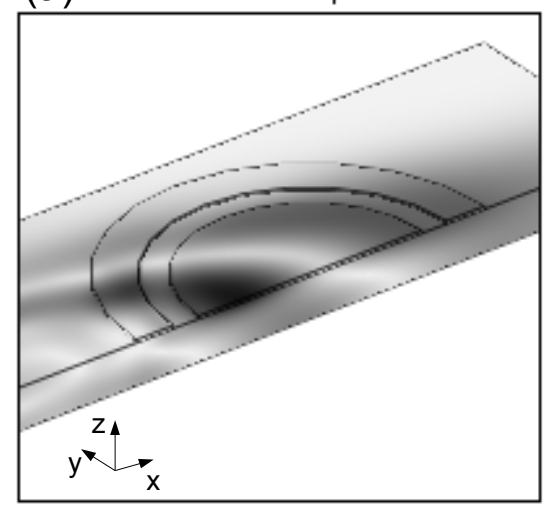

Max: 2,30e-12

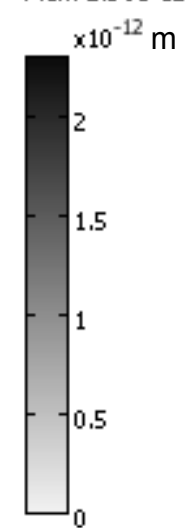

(b)

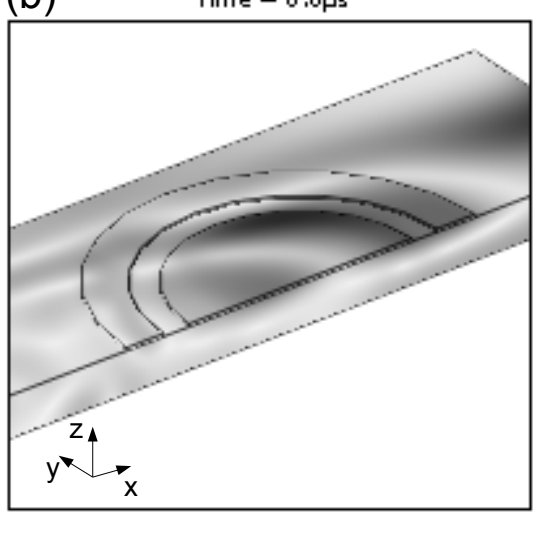

Max: 2.30 e- 12

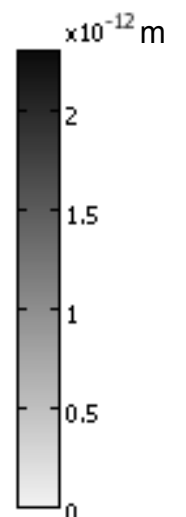

Fig. 11: Temporal development of the magnitude of the displacement vector at arrival time of the $S_{0}$ plate wave mode at the sensors position for times $t=5.0 \mu \mathrm{s}(\mathrm{a})$ and $6.0 \mu \mathrm{s}(\mathrm{b})$.

In the following we want to investigate the influence of displacement direction, displacement amplitude, excitation time and position of the model source within the specimen on the simulated acoustic emission signals in our model specimen.

To verify the quality of this model setup, we want to compare the obtained simulated signals to real experimental data signals recorded during four-point-bending experiments.

\section{Experimental setup and pattern recognition method}

For comparison with results of simulation, acoustic emission signals were recorded during bending experiments of CFRP specimens. The four-point-bending tests were conducted according to DIN-EN-ISO 14125 with a deformation rate $v=0.01 \mathrm{~mm} / \mathrm{s}$ and support distances like shown in figure 12. The used specimens consist of ten unidirectional Hexply T800/913 plies, cut into dimensions of $100 \mathrm{~mm} \times 15 \mathrm{~mm} \times$ $1.4 \mathrm{~mm}$ (length $\mathrm{x}$ width $\mathrm{x}$ height). The acoustic emission signals were recorded using a Physical Acoustic PCl-2 system with two sensors of type WD in linear geometry at an acquisition rate of $10 \mathrm{MS} / \mathrm{s}$. To suppress detection of friction noise a band-pass filter was used ranging from $20 \mathrm{kHz}$ to $1 \mathrm{MHz}$. An additional band-pass filter is included within the preamplifier ranging from $80 \mathrm{kHz}$ to $2 \mathrm{MHz}$. After waveform acquisition, the signals source position was determined based on a hyperbolic location technique using the linear sensor geometry shown in figure 12.

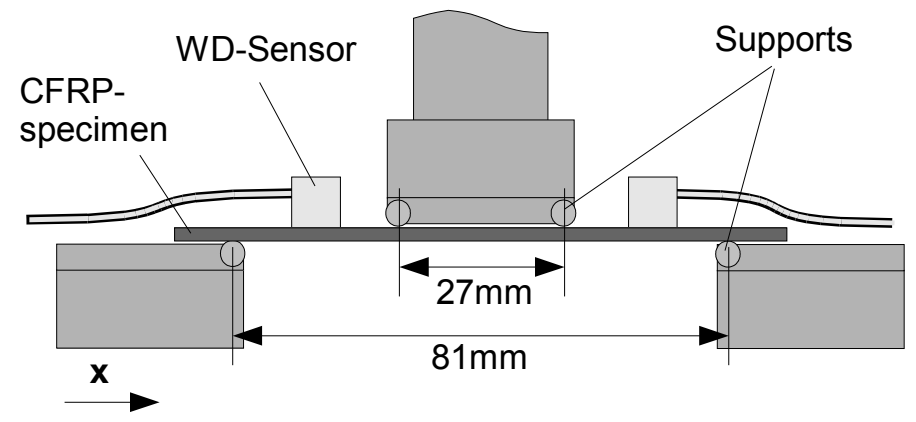

Fig. 12: Bending test specification according to DIN-EN-ISO 14125 with two sensors of type WD attached to the specimen.

In order to distinguish different failure types, a pattern recognition approach was used, similar to those described in more detail in earlier works $[25,26]$. Here we give a short review and introduce some modifications to this approach. 
To adapt the established pattern recognition methodology to discriminate acoustic emission signals corresponding to several failure mechanisms in CFRP, we use the frequency parameters extracted from the recorded waveforms, as summarized in table 2.

\begin{tabular}{|l|l|}
\hline Feature & Definition \\
\hline Average Frequency $[\mathrm{Hz}]$ & $\langle f\rangle=N / T$ with $N$ : Number of threshold crossings and $T$ : duration \\
& of signal \\
\hline Weighted Peak-Frequency $[\mathrm{Hz}]$ & $\left\langle f_{\max }\right\rangle=\sqrt{f_{\max } \cdot \int f \cdot \hat{U}(f) d f / \int \hat{U}(f) d f}$ with \\
& $f_{\max }$ : frequency position of the maximum frequency intensity \\
& $\hat{U}(f)$ : Fourier-Transformation of $U(t)$ \\
\hline Partial Power 1-3 [\%] & $\int_{2} \hat{U}^{2}(f) d f / \int_{0 k \mathrm{kz}}^{1200 \mathrm{kHz}} \hat{U}^{2}(f) d f$ \\
& $\int_{f_{1}}$ \\
& Partial Power $1: \mathrm{f}_{1}=0 \mathrm{kHz} ; \mathrm{f}_{2}=200 \mathrm{kHz}$ \\
& Partial Power $2: \mathrm{f}_{1}=200 \mathrm{kHz} ; \mathrm{f}_{2}=350 \mathrm{kHz}$ \\
& Partial Power $3: \mathrm{f}_{1}=350 \mathrm{kHz} ; \mathrm{f}_{2}=1200 \mathrm{kHz}$ \\
\hline
\end{tabular}

Table 2: Waveform features used for pattern recognition method.

The extracted features were normalized using their variance and are projected to their principal components axis. Finally, the datasets were classified using the cluster algorithm k-means available in the software package Noesis used for the pattern recognition process [27].

To evaluate the numerical grade of discrimination we calculated the parameters $R$ and $\tau$ as defined by Davies-Bouldin and Tou [28, 29]:

$R_{i j}=\frac{D_{i}+D_{j}}{D_{i j}}, \quad i, j=1, \ldots, C ;$

$r_{i}=\max \left(R_{i j}\right) \quad \forall i \neq j, \quad i, j=1, \ldots, C ;$

$R=\frac{1}{C} \sum_{i=1}^{C} r_{i}$;

$\tau=\frac{\min \left(D_{i j}\right)}{\max \left(D_{k}\right)} ; \quad i, j, k=1, \ldots, C ;$

Here $D_{i}$ and $D_{j}$ are defined as the average distance between members within a cluster and $D_{i j}$ as the distance between the respective cluster centers. The criterion $R$ is then calculated from the maximum values of $R_{i j}$ divided by the cluster numbers $C$. The criterion $\tau$ is calculated from the minimal distance $\min \left(D_{i j}\right)$ between members of clusters $i$ and $j$ and the maximum distance $\max \left(D_{k}\right)$ of members within cluster $k$. Therefore $R$ is a measure for the average compactness of all clusters and $\tau$ is a measure for the mean spatial distribution of the clusters relative to each other. Hence the cluster members separate more distinctly for low values of $R$ and high values of $\tau$.

To obtain the distinguishable number of acoustic emission waveform types we followed an approach established by Anastassopoulos et al. [30], that was already successfully applied to similar classification problems [25, 26]. The result of such an investigation is shown in figure 13 for one representative measurement. In the investigated specimens the $R, \tau$ and $R / \tau$ Ratio criteria clearly suggest a separation into three types of waveforms. 


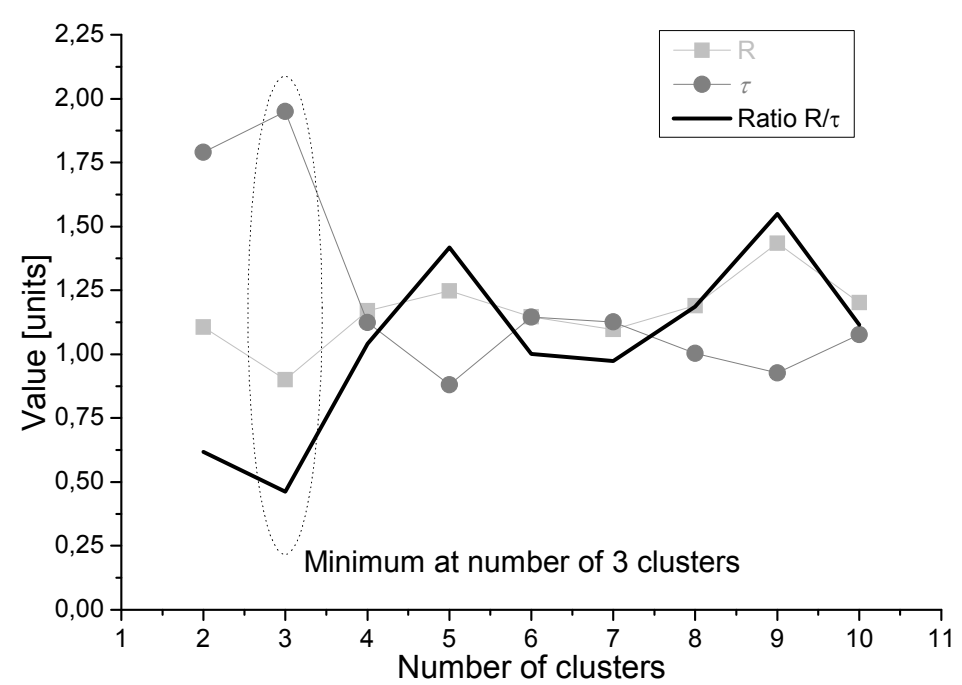

Fig. 13: Investigation of a representative CFRP-specimen using pattern recognition. The dependency of the criteria $R, \tau$ and $R / \tau$ on the number of clusters suggests a separation into three types of acoustic emission signals.

We attribute these three types of signals to, matrix cracking, fiber-matrix interface failure and fiber-breakage, respectively. This attribution is based on frequency spectra characteristics (see also section 5), as reported by various authors [1, 2, 3, 4, 5]. In addition we applied scanning electron and ultrasonic microscopy to correlate the preferential positions of the acoustic emission signals belonging to one cluster with the respective failure mechanism.

\section{Discussion of source excitation parameters}

To evaluate the correlation between the microscopic failure mode and the macroscopically detected acoustic emission signal the influence of the input parameters on the simulation was investigated.

To this end we varied the source excitation time $T_{e,(x y z)}$, the direction of displacement and the displacement amplitude $\mathrm{d}_{(\mathrm{xyz})}$. Finally we investigated the changes of the simulated acoustic emission signals if the vertical position of the acoustic emission source model changes within the plate. In order to compare the influence of these parameters on the frequency spectrum we calculate the sensors surface velocity response signal $\mathrm{S}(\mathrm{t})$ as described in section 2 and calculate the corresponding FastFourier-Transformation (FFT) using the Daniel-Lanczos algorithm with a rectangular window function. To compare the frequency spectra of different calculations we normalized the resulting magnitude-spectra (defined as $|\hat{U}(f)|$ ) to their absolute value, to take into account the effect of different source excitation energies.

\section{a) Variation of excitation time}

As already pointed out by Giordano et al. the frequency spectra of acoustic emission signals are strongly influenced by the elastic properties of the material and the ratio between the relaxation time of the (viscoelastic) material and the excitation time given by the crack progress [9]. In our case the elastic properties are those of resin in the case of matrix cracks and the elastic properties of carbon fiber in the case of fiber breakage. In figure 14 and 15 the effect of the different elastic properties of these materials on the frequency spectra are shown for an excitation in $\mathrm{x}$-direction for 
various excitation times. A distinct difference in frequency spectra of matrix cracking (high contribution at low frequency range) and fiber breakage (high/dominant contribution at high frequency range) is observed in the simulated frequency spectra.

These differences in frequency composition reflect the change of the ratio between excited antisymmetric and symmetric plate-wave modes. In our simulation it was observed, that for "fiber-breakage" the stimulation of $\mathrm{S}_{0}$-modes dominates, while the $\mathrm{A}_{0}$-mode contributions superimposed by boundary reflections dominate in case of "matrix-cracking". For our CFRP-specimens, the propagating $\mathrm{S}_{0}$-mode contains higher frequency contributions and therefore the detected frequency spectra show dominant contributions at frequencies above $450 \mathrm{kHz}$. In contrast, the propagating $\mathrm{A}_{0}$-mode contains lower frequencies. This result of the simulation is in agreement with experimental observations of various authors $[1,2,3,4,5]$.

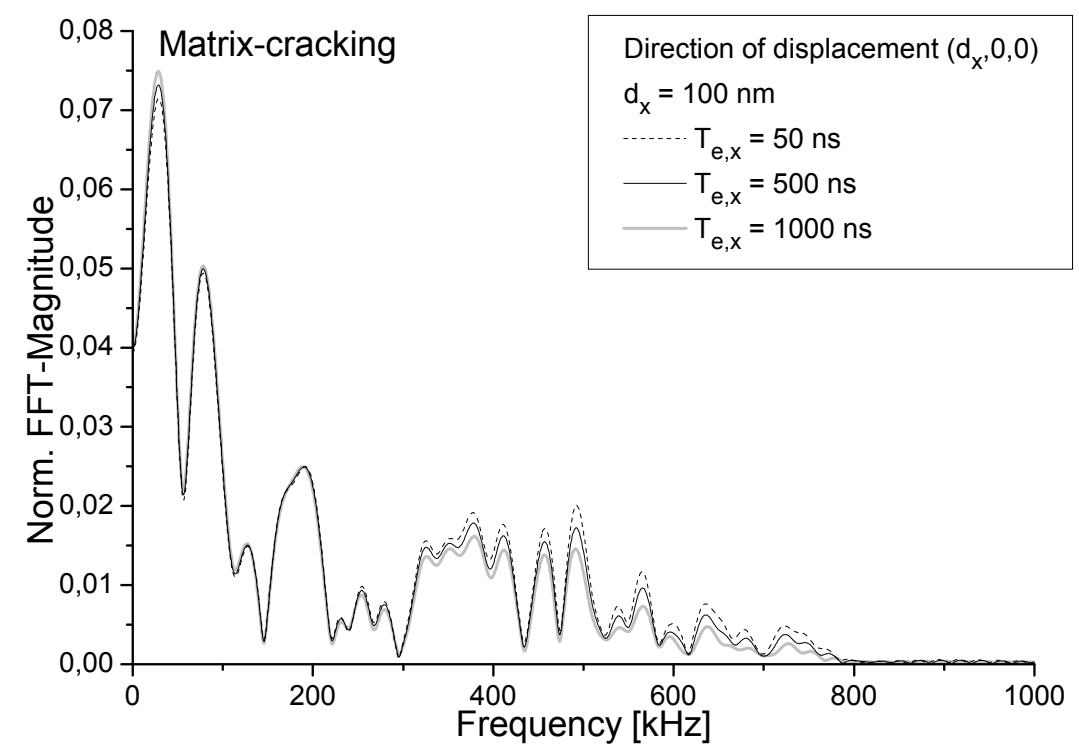

Fig. 14: Influence of excitation time $T_{e, x}$ for displacement in $x$-direction with $d_{x}=100 \mathrm{~nm}$ for resin elastic properties.

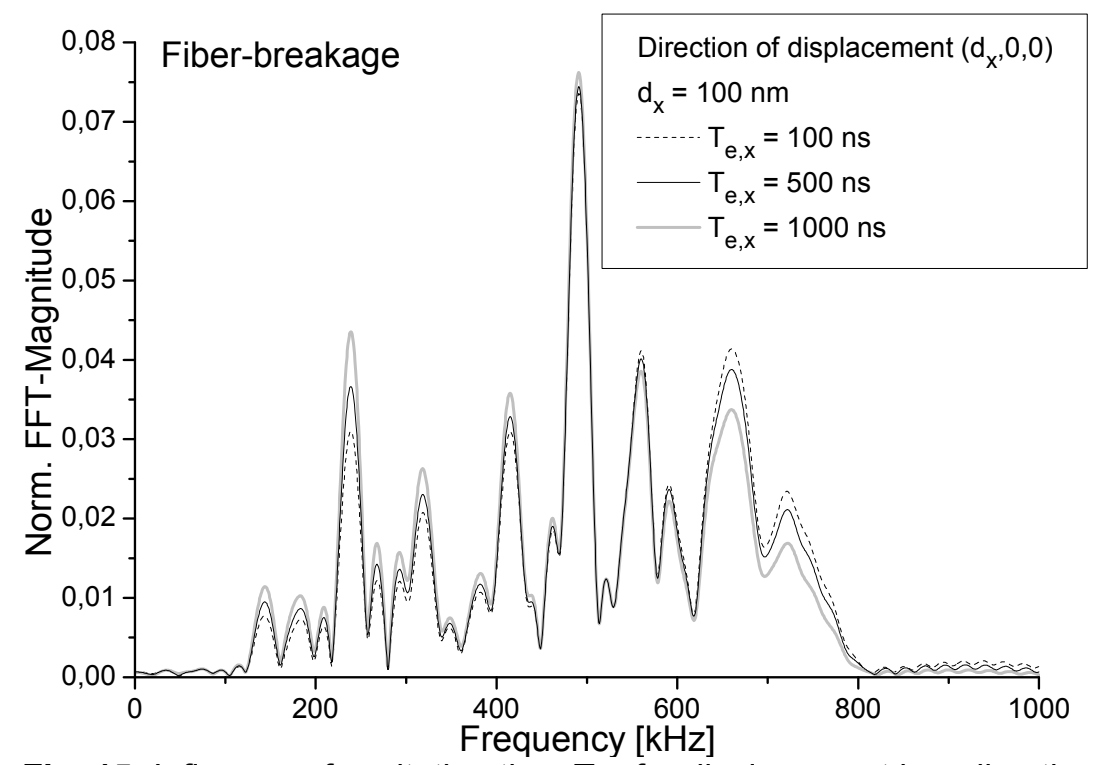

Fig. 15: Influence of excitation time $T_{e, x}$ for displacement in $x$-direction with $d_{x}=100 \mathrm{~nm}$ for fiber elastic properties. 
In addition, a small influence of the source excitation time $T_{e, x}$ is observed. When $T_{e, x}$ increases (i.e. the crack surface is displaced more slowly) the frequency content shifts to lower frequencies, which results from a shift of energy contributions to the $\mathrm{A}_{0}$-mode. The same effect was observed in resin for excitation in z-direction (not shown) which demonstrates that the spectral changes resulting from different excitation times do not depend on the chosen source radiation direction.

\section{b) Direction of displacement}

In general the frequency content of acoustic emission signals will depend on the direction of displacement relative to the out-of-plane direction of the plate.

As already described in section 2, specific failure mechanisms in fiber reinforced structures naturally result in acoustic emission sources with specific oriented source radiation directions. To this end we investigated the influence of the displacement direction within our crack model for matrix cracking on the frequency spectra of the acoustic emission signals. The result of a simulation with equal excitation time $T_{e}=$ $100 \mathrm{~ns}$ and equal displacement amplitude $d=100 \mathrm{~nm}$ is shown in figure 16 . The simulated frequency spectra show in general good agreement. Only small differences are observed for frequencies above $400 \mathrm{kHz}$. In comparison to experimental data of transverse matrix cracking reported by Prosser et al. [35] this result is unexpected. As discussed before the strong low frequency parts suggest a strong $A_{0}$-mode contribution. This is only partially the case, since the $A_{0}$-mode is superimposed by the reflections from the boundaries. In addition the stacking sequence of the CFRPspecimen is a major factor of influence on the observed frequency spectra. Comparative simulations of a layup with $0 / 90^{\circ}$ stacking order show less intense low frequency parts. This is a result of the additional vertical anisotropy introduced by variations in the stacking sequence of the CFRP. For the purely unidirectional CFRPspecimens in the present investigation the frequency content is instead independent of the direction of displacement.

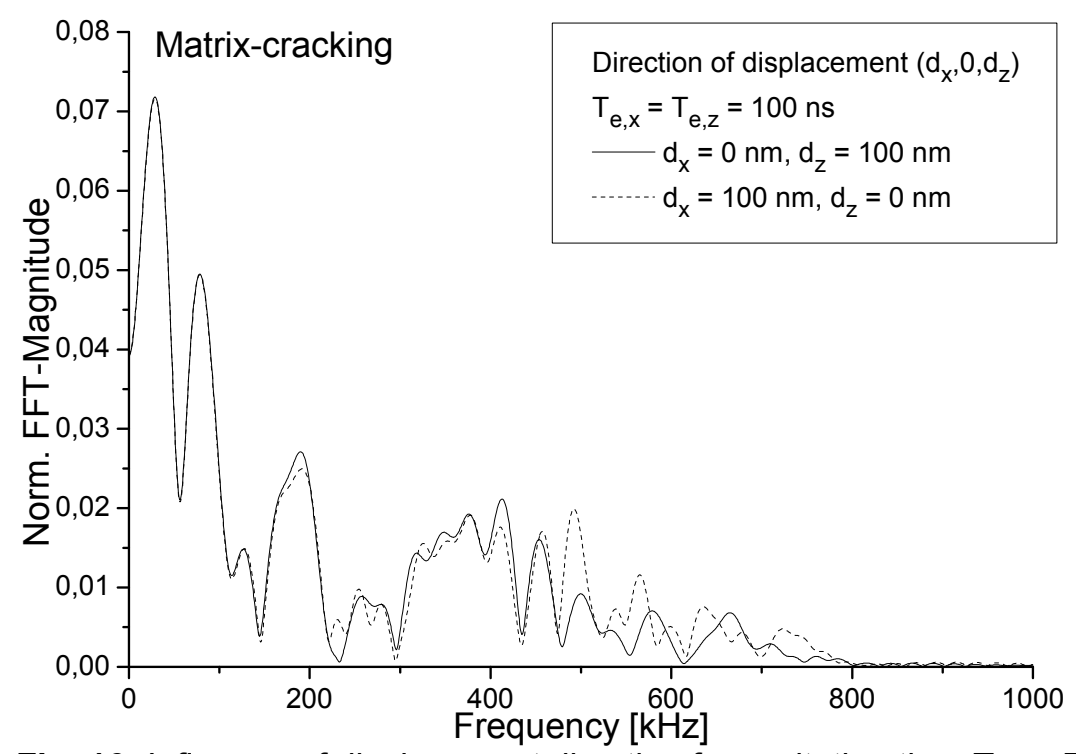

Fig. 16: Influence of displacement direction for excitation time $T_{e, x}=T_{e, z}=100 \mathrm{~ns}$ and displacement in $x$ or z-direction with $d_{x}=d_{z}=100 \mathrm{~nm}$.

The influence of the displacement direction was not investigated for fiber breakage since this is not expected to happen in the out-of-plane direction (z-direction) of a fiber reinforced plate structure. A systematic investigation of frequency spectra 
obtained from simultaneous excitation in $\mathrm{x}$ - and $\mathrm{z}$-direction is described in section 5 since such excitations are attributed to fiber-matrix interface mechanisms.

\section{c) Variation of excitation amplitude d}

The excitation amplitude $d$ of the displacement determines the energy content of the failure mechanism and the respective acoustic emission signal. A common approach to correlate acoustic emission signals with failure mechanisms makes use of this energy content. This approach is only valid if the size of the acoustic emission source and the individual energy release show narrow distributions. In the case of carbon fibers, this can be expected to be fulfilled, as long as only single fiber breakage occurs. In the case of matrix cracking, the size of the crack surface increments shows a broad distribution. In this case we will show below, that frequency spectra characterize the respective failure mechanism better than the energy content.

The simulation for matrix-cracking with excitation in $\mathrm{x}$-direction, excitation time $\mathrm{T}_{\mathrm{e}}=$ 100 ns and varying displacement amplitude is shown in figure 17 . The simulated relative frequency composition is independent of the displacement amplitude. Moreover, the same effect was obtained for fiber-breakage in x-direction and matrixcracking in z-direction.

Hamstad et al. observed a similar behavior simulating dipole-type point sources in isotropic material. They observed that the ratio of $S_{0^{-}}$and $A_{0^{-}}$-magnitude is independent of the acoustic emission source strength [16].

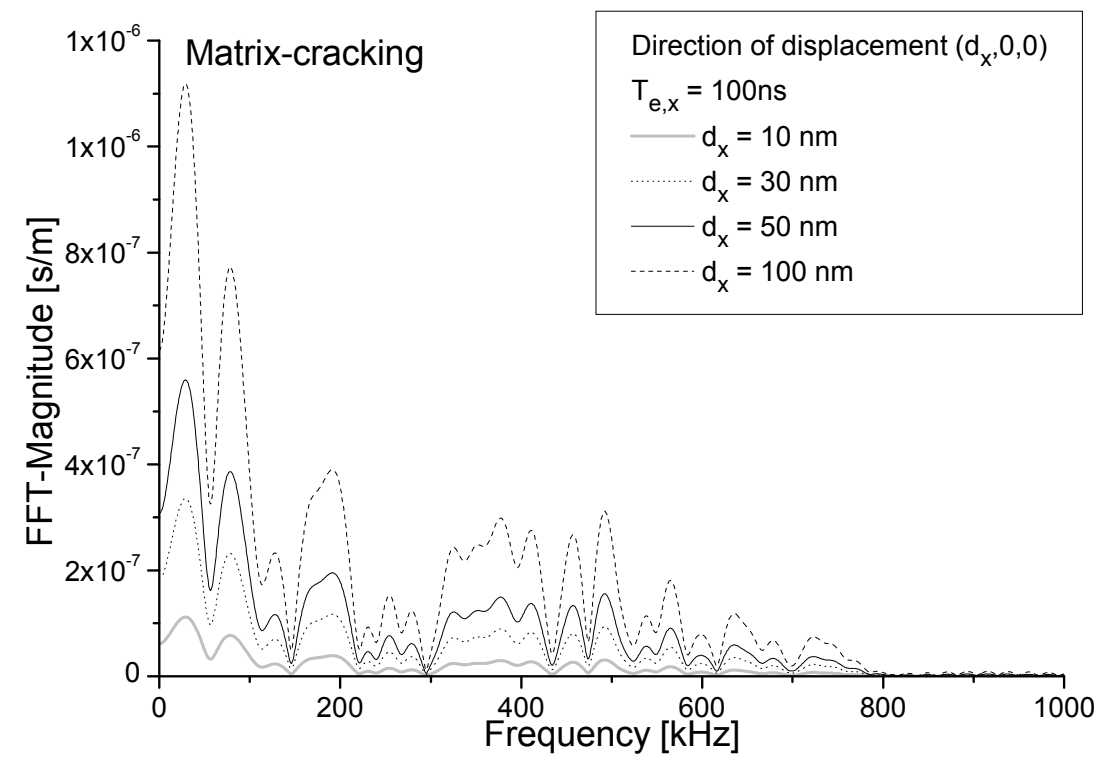

Fig. 17: Variation of the displacement amplitude $d_{x}$ at constant excitation time $T_{e, x}=100 \mathrm{~ns}$.

\section{d) Dependence on acoustic emission source position}

As already pointed out by Hamstad et al., the z-position of the acoustic emission source is crucial for the ratio of stimulated plate wave modes [16]. So far our results were obtained for a position symmetrically centered with respect to all three axes (center-point of the used specimen). In order to use frequency criteria obtained from acoustic emission waveforms to distinguish different failure mechanisms, only a limited dependence of the frequency spectra on the sources z-position is acceptable.

Figures 18 and 19 show simulations of frequency spectra resulting from matrixcracking and fiber-breakage in x-direction located at four different z-positions as marked in figure 3 . The $(x, y)$-position, the excitation time $T_{e x}=100 \mathrm{~ns}$ and the 
displacement amplitude $d_{x}=100 \mathrm{~nm}$ were kept constant. The position $\mathrm{z}=0.66 \mathrm{~mm}$ locates the source practically at the specimen surface, since the source model's $z$ length is $100 \mu \mathrm{m}$.

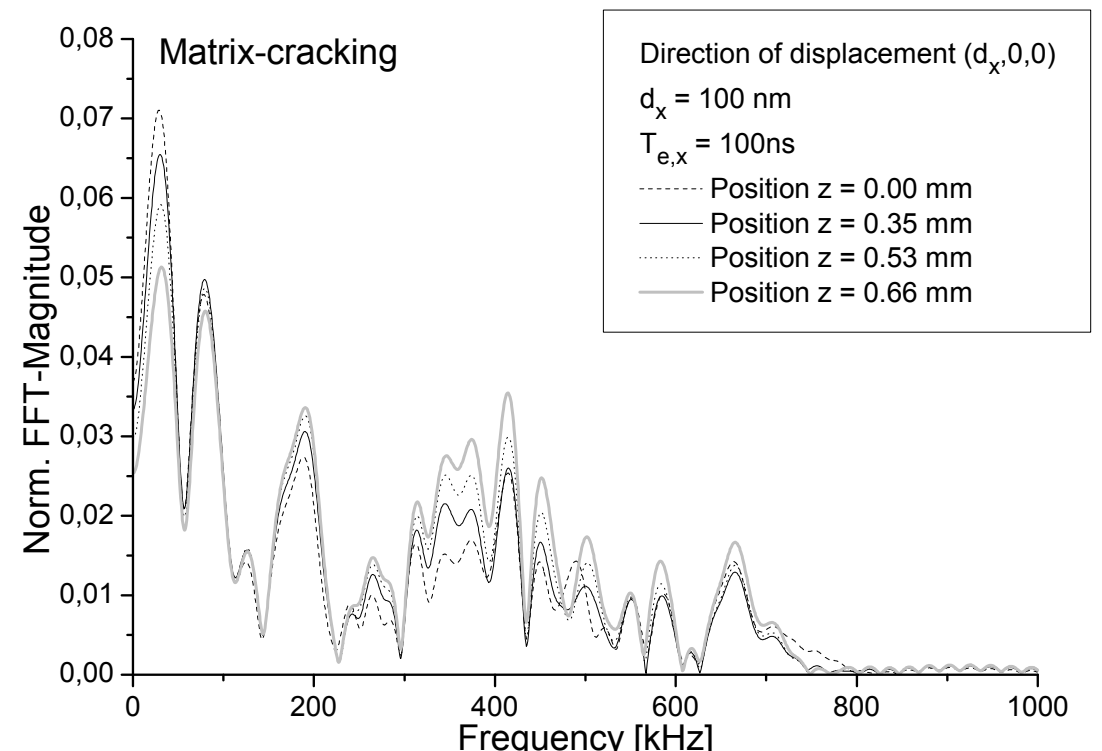

Fig. 18: Effect of variation of the z-position of the acoustic emission source model within the plate. Normalized frequency spectra of four different z-positions for matrix-cracking with excitation time $\mathrm{T}_{\mathrm{e}, \mathrm{x}}=100 \mathrm{~ns}$ and displacement $\mathrm{d}_{\mathrm{x}}=100 \mathrm{~nm}$.

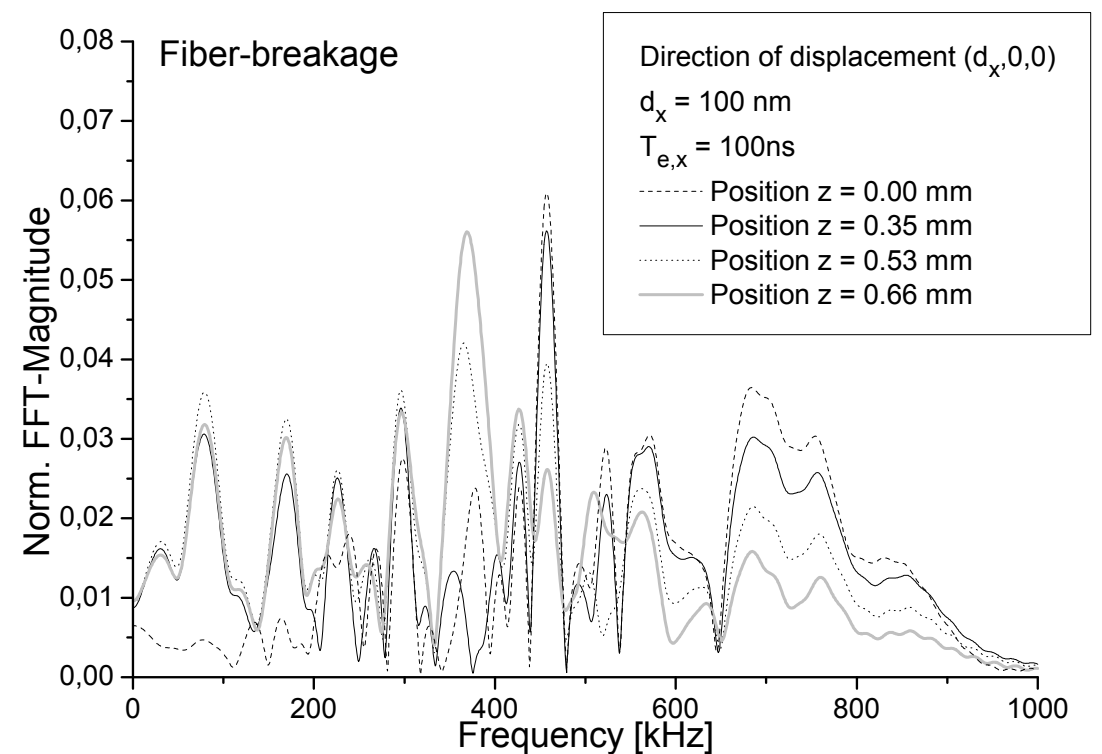

Fig. 19: Effect of variation of the z-position of the acoustic emission source model within the plate. Normalized frequency spectra of four different z-positions for fiber-breakage with excitation time $T_{e, x}=100 \mathrm{~ns}$ and displacement $d_{x}=100 \mathrm{~nm}$.

In the case of matrix cracking a significant influence of the source's z-position is found, which is most pronounced at the position $z=0.66 \mathrm{~mm}$. With increasing $z-$ position the spectral weight below $200 \mathrm{kHz}$ decreases, while the spectral weight around $400 \mathrm{kHz}$ increases. For fiber-breakage (shown in figure 19) the influence of the source position is even more pronounced. The spectral contributions above $400 \mathrm{kHz}$ decrease significantly with increasing z-position, while the contributions around $400 \mathrm{kHz}$ increase. 
Despite the shifts in the weight of the frequency distribution the spectra remain characteristic for matrix-cracking and fiber-breakage, respectively. A quantitative comparison for the investigated source positions is summarized in table 3 . Clearly frequency parameters are still valid parameter to distinguish between matrix-cracking and fiber-breakage.

\begin{tabular}{|l|l|l|l|}
\hline z-Position & Feature & Fiber-breakage & Matrix-cracking \\
\hline \multirow{2}{*}{$z=0.00 \mathrm{~mm}$} & Partial Power 3 & $86.6 \%$ & $29.0 \%$ \\
\cline { 2 - 4 } & weighted Peak-Frequency & $524 \mathrm{kHz}$ & $187 \mathrm{kHz}$ \\
\hline \multirow{2}{*}{$\mathrm{z}=0.35 \mathrm{~mm}$} & Partial Power 3 & $81.5 \%$ & $34.4 \%$ \\
\cline { 2 - 4 } & weighted Peak-Frequency & $496 \mathrm{kHz}$ & $190 \mathrm{kHz}$ \\
\hline \multirow{2}{*}{$\mathrm{z}=0.53 \mathrm{~mm}$} & Partial Power 3 & $73.7 \%$ & $39.6 \%$ \\
\cline { 2 - 4 } & weighted Peak-Frequency & $495 \mathrm{kHz}$ & $209 \mathrm{kHz}$ \\
\hline \multirow{2}{*}{$\mathrm{z}=0.66 \mathrm{~mm}$} & Partial Power 3 & $64.8 \%$ & $41.7 \%$ \\
\cline { 2 - 4 } & weighted Peak-Frequency & $439 \mathrm{kHz}$ & $212 \mathrm{kHz}$ \\
\hline
\end{tabular}

Table 3: Calculated features as defined in table 2 of simulated fiber breakage and matrix cracking signals with different z-positions.

The strong dependence of the frequency distribution on the source position shows, that the distinction of these failure mechanisms is not possible based on one single frequency based criteria like, e.g. the maximum peak frequency. Instead the classification should rely on several characteristic frequency based criteria, like FFTmagnitude contributions in different given frequency intervals or similar descriptors derived from wavelet-transformations.

\section{Comparison of simulation results with experimental signals}

To demonstrate the quality of the presented simulation, the simulated acoustic emission signals are compared to experimental data. As described in section 3 the signals were recorded during four-point bending experiments on unidirectional CFRP specimens. The signals used here were localized approximately at the position $x=0 \mathrm{~mm}$ but undefined $(y, z)$-position. This means that the experimental signals have a propagation distance comparable to the simulated signals. The experimental waveforms were assigned to a particular failure mechanism using the pattern recognition approach explained in section 3. To compare simulated with experimental signals we calculate the wavelet transformation using the software package AWARE++ and discuss the obtained wavelet-transformation (WT) diagrams [31]. In contrast to the simulated signals, the experimental acoustic emission signals are modified due to several built-in band-pass filters in the electronic acquisition system. To obtain simulated signals comparable to our experimental setup, we applied a high-pass filter with cut-off frequency at $80 \mathrm{kHz}$ to the simulated results.

In general, deviations between simulated and experimental signals can be attributed to two different mechanisms not included within the model.

First, the used sensor has a frequency dependent sensitivity which is not completely linear within the relevant frequency range. This can emphasize the intensity at the sensors resonant-like frequencies as observed around $200 \mathrm{kHz}$ (right panel of figure 20).

Secondly, the path of wave propagation has an influence on the detailed shape of the signal. Such an influence can occur due to pre-existing cracks within the propagation path, or deviations between the model and the real source position.

A comparison between the simulation of matrix cracking with excitation in x-direction $\left(T_{e, x}=1000 \mathrm{~ns}, d_{x}=100 \mathrm{~nm}\right)$ and an experimental signal is displayed in figure 20 . For 
both diagrams low frequency parts below $200 \mathrm{kHz}$ dominate the signal which are attributed to a $\mathrm{A}_{0}$-mode superimposed by the reflections from the adjacent boundaries. The stronger contributions below $50 \mathrm{kHz}$, as visible in the FFT-spectra of figures 14, 16, 17 and 18 are suppressed by the high-pass filter introduced to simulate the experimental conditions. With this chosen filter setup the simulated signal agrees systematically with the experimental signal.
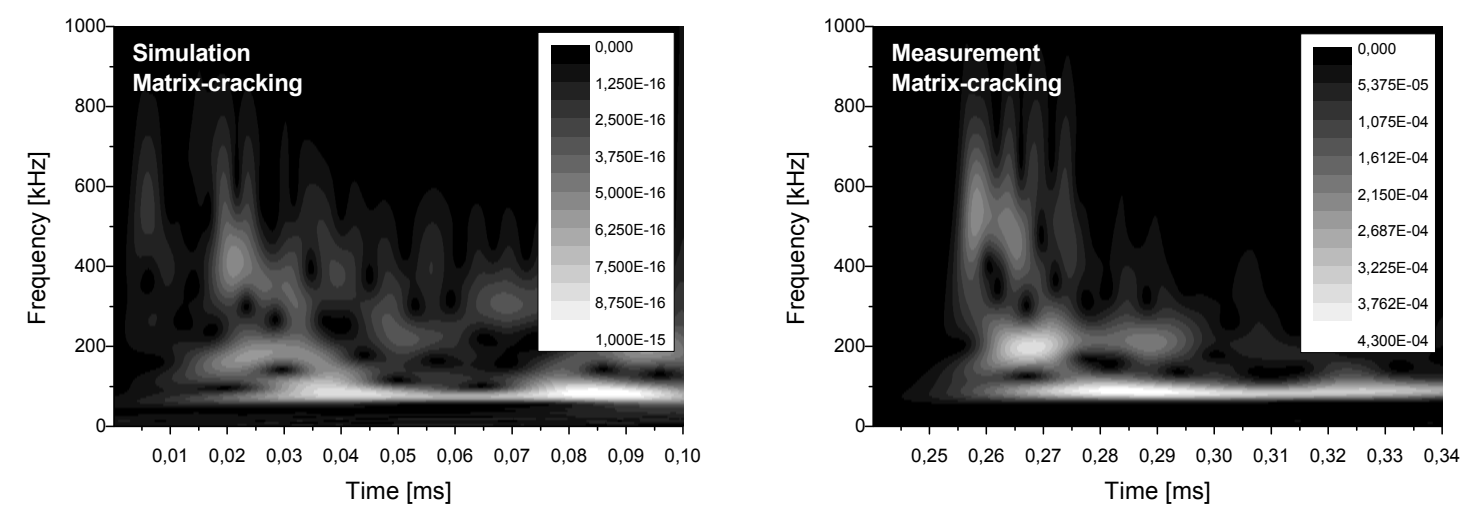

Fig. 20: Simulation of matrix cracking with excitation time $T_{e, x}=1000$ ns and displacement in $x-$ direction $d_{x}=100 \mathrm{~nm}$ (left) and experimentally observed signal (right).

Figure 21 shows the comparison between a simulated fiber-breakage and an experimentally obtained fiber-breakage signal. Both wavelet-diagrams show good agreement in their overall temporal evolution of frequency contributions. The $\mathrm{S}_{0}$ mode is dominant as seen by the high frequency contributions at the beginning of the time axis. As already discussed in section 2, the sensor of type WD contains two piezoelectric elements. The fact that the acoustic emission signal is detected earlier at the ring-element than at the disc-element results in a double feature of the $\mathrm{S}_{0}$ mode that is observed in both wavelet-diagrams.
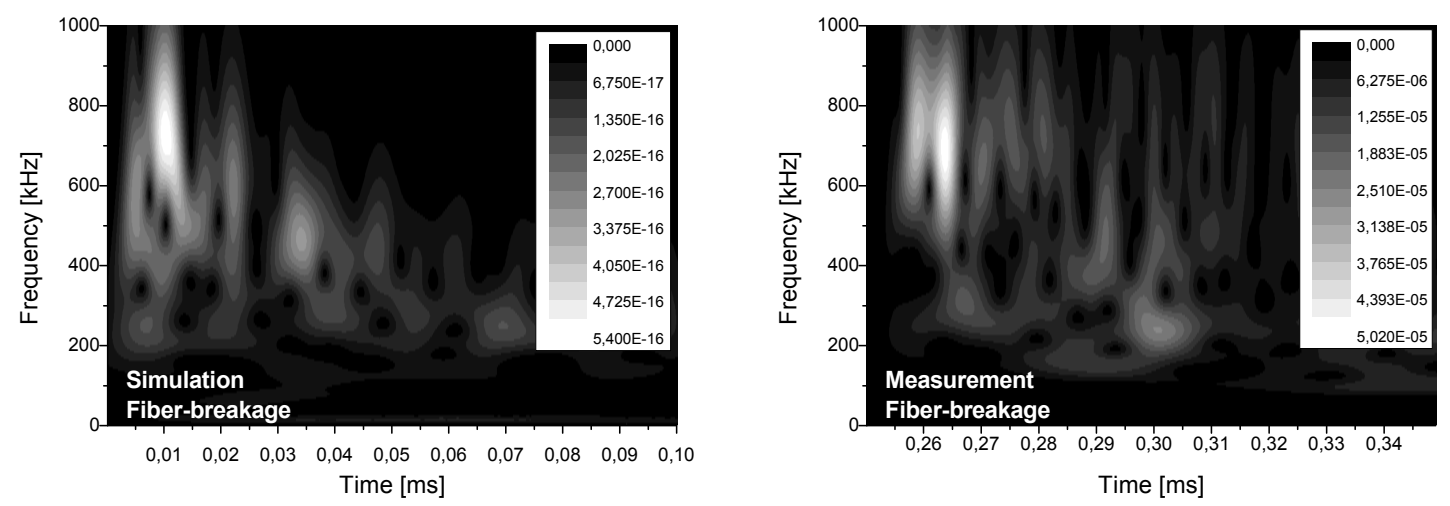

Fig. 21: Simulation of fiber-breakage with excitation time $T_{e, x}=50 \mathrm{~ns}$ and displacement in $\mathrm{x}$-direction $\mathrm{d}_{\mathrm{x}}=100 \mathrm{~nm}$ (left) and experimentally observed signal (right).

In the case of interface failure a short stimulation of the carbon fiber with low displacement amplitude in $\mathrm{x}$-direction is expected. Therefore we expect a lower displacement in $x$-direction $\left(d_{x}=5 \mathrm{~nm}\right.$ to $\left.10 \mathrm{~nm}\right)$ than for fiber-breakage. For the displacement in $z$-direction we assumed values comparable to the case of matrixcracking $\left(d_{z}=100 \mathrm{~nm}\right)$ occurring simultaneously with the displacement in $\mathrm{x}$-direction.

The simulated signal of a fiber-matrix interface failure is compared to experiment in figure 22. The simulated and experimental signals show more complex waveletdiagrams than fiber-breakage or matrix-cracking. The strongest frequency 
contribution is observed around $200 \mathrm{kHz}$. At the beginning of the time axis additional contributions arise from the excitation of the $S_{0}$-mode. A contribution in the intermediate frequency range between $300 \mathrm{kHz}$ and $600 \mathrm{kHz}$ is observed over a broad time interval. With the chosen excitation parameters a good match between simulated and experimental signals was found.
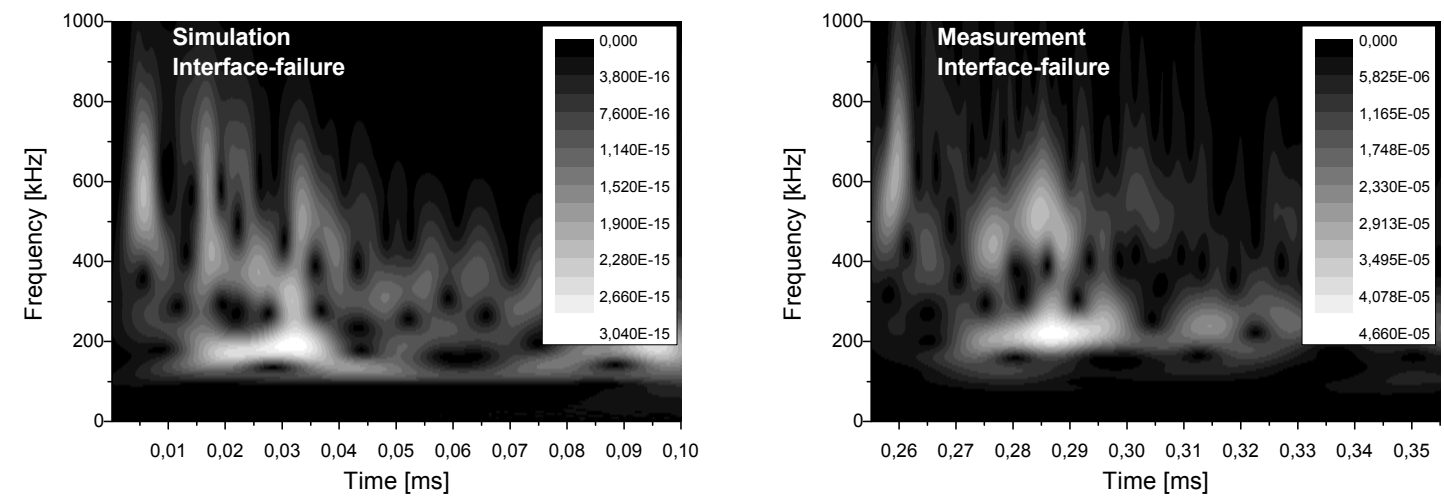

Fig. 22: Simulation of fiber-matrix interface failure with excitation time $T_{e, x}=500 \mathrm{~ns} T_{e, z}=1000 \mathrm{~ns}$, displacement in $x$-direction $d_{x}=5 \mathrm{~nm}$ and displacement in z-direction $d_{z}=100 \mathrm{~nm}$ (left) and experimentally observed signal (right).

Figure 23 shows the simulated frequency spectra of a systematic variation of the ratio between the displacement components directed in $\mathrm{x}$ - and $\mathrm{z}$-direction. The ratio of the displacement amplitude in $x$ - and $z$-direction influences the frequency spectrum of the simulated acoustic emission signal significantly. With increasing displacement in x-direction, the contributions at frequencies above $400 \mathrm{kHz}$ increases. This corresponds to a stronger excitation of the $\mathrm{S}_{0}$-mode. The displacement in x-direction depends on the shear stress between fiber and matrix at the start of debonding. A higher adhesion strength between fiber and matrix means a higher expected shear stress, which translates in a higher displacement component along the fiber axis (x-direction). This suggests that the intensity of the excitation of the $S_{0}$-mode correlates with the fiber-matrix adhesive strength, i. e. the higher the $S_{0^{-}}$ mode intensity, the higher the adhesive strength.

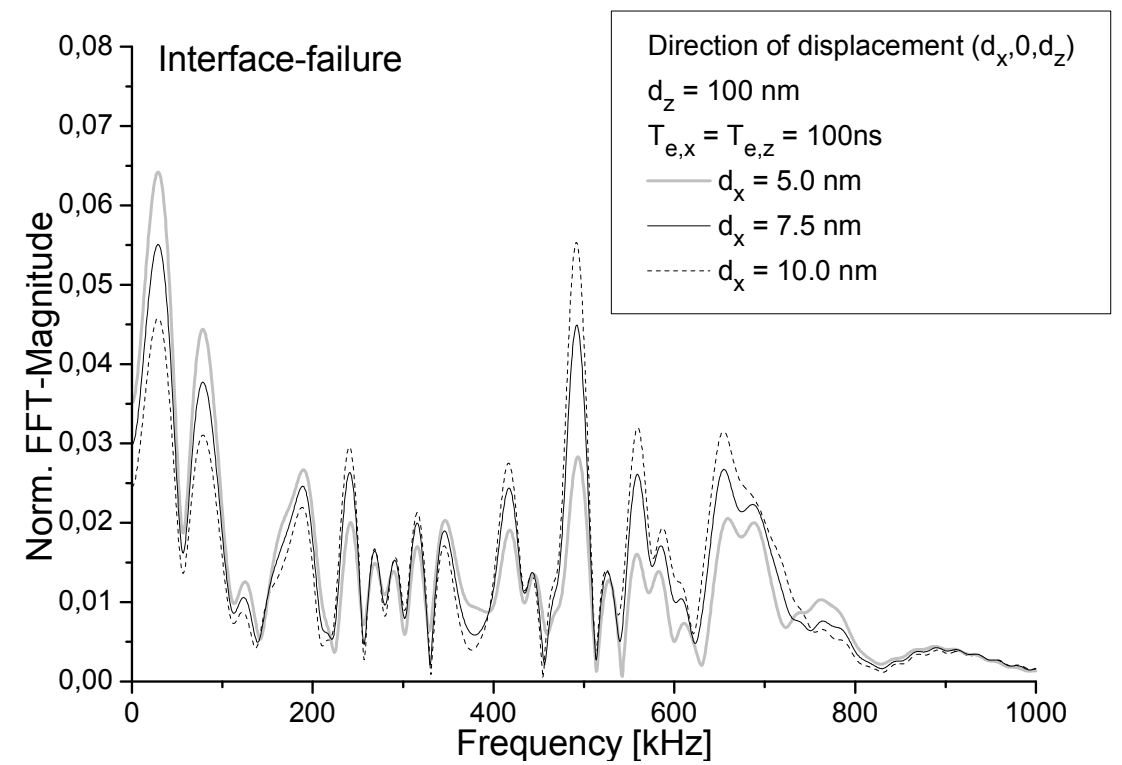

Fig. 23: Influence of the ratio between displacement components directed in $x$ - and z-direction. The excitation times are $T_{e, x}=T_{e, z}=100 \mathrm{~ns}$, the displacement amplitude in z-direction is $d_{z}=100 \mathrm{~nm}$ and $d_{x}$ is varied from $5.0 \mathrm{~nm}$ to $10.0 \mathrm{~nm}$. 
The simulated frequency spectra corresponding to interface failure show some resemblance to those of fiber-breakage signals if, in the latter case, the source position is close to the plate surface. But in contrast the signals corresponding to interface failure show stronger contributions for frequencies below $200 \mathrm{kHz}$. As a consequence the signals can still be distinguished from fiber-breakage signals. On the other hand, the distinction to matrix-cracking is based on stronger contributions of the interface failure signals for frequencies above $450 \mathrm{kHz}$.

A quantitative comparison of values obtained from the experimental data and the simulated signals is shown in figure 24. The plot of Partial-Power 3 over weighted Peak-Frequency as defined in table 2 is used to separate the different failure mechanisms due to their differences in frequency content. The value ranges for simulated fiber breakage and matrix cracking compare well to the experimental data. In contrast, the simulated datapoints of interface failure are not very close to the mean experimental values but still within the range of experiment. Since interfacial failure is attributed to various failure mechanisms, the presented model source may not fully represent them. In addition deviations between simulation and experiment can result from runtime differences between experimental signals and simulated signals. This is caused by the unknown $(y, z)$ source position of the experimental signals which is not directly comparable to the position of the simulated signals, which were obtained for $(x, y, z)=(0,0,0)$.

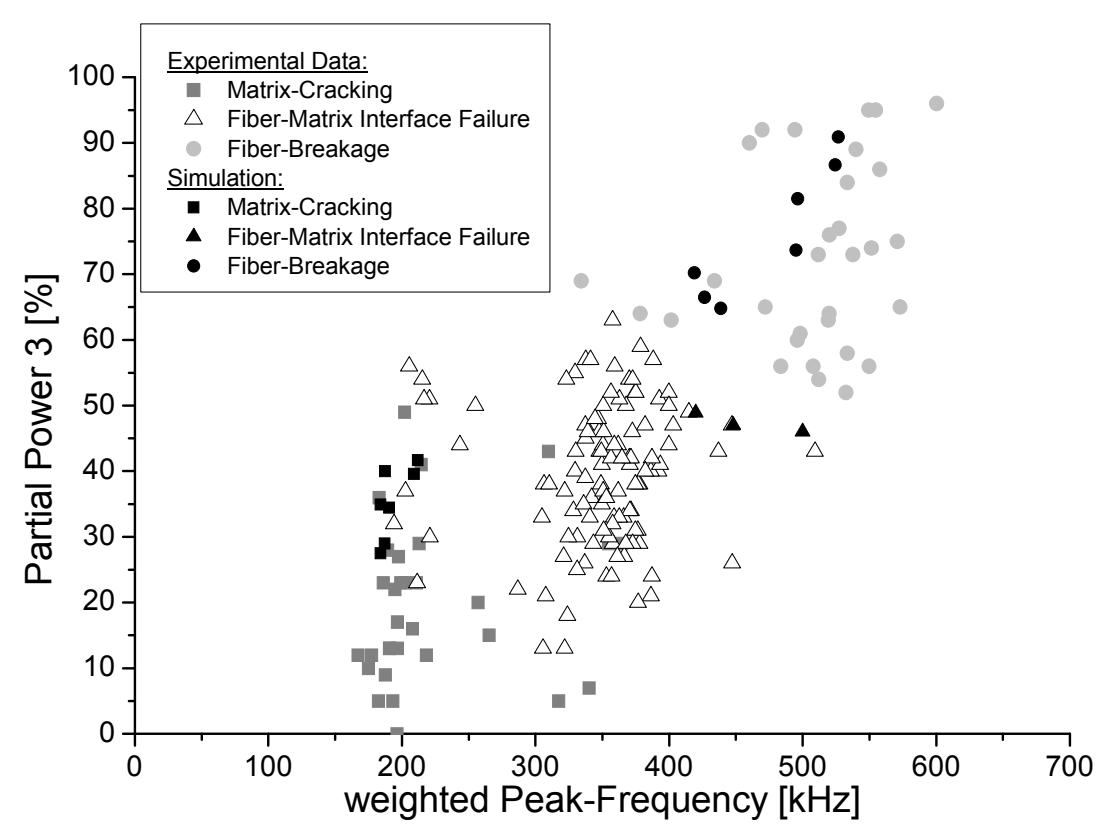

Fig. 24: Scatter plot of the weighted peak frequency over partial power 3 of the respective classification into matrix cracking, fiber-matrix interface failure and fiber-breakage obtained from the pattern recognition approach compared with results from the finite element simulations.

\section{Summary}

The acoustic emission signals excited by the presented source model in combination with the specimen geometry results in plate waves that propagate within the model specimen. The source model is solely based on a linear displacement of the crack surface and does not use vibrating acoustic emitters or driven forces as source mechanism. To compare the simulated acoustic emission signals with experiments, 
the aperture effect of the used acoustic emission sensor had to be taken into account, since this significantly influences the shape and frequency content of the detected waveforms. The acoustic emission signals that are simulated using the presented specimen-sensor interaction model describe the experimentally obtained signals measured in corresponding geometry. In particular, the excited frequency range and temporal evolution of the simulated acoustic emission signals show systematic agreement with experiment.

The response of the model to an increased excitation time is expressed in a shift of the frequency weight to lower frequencies. This result is qualitatively comparable to analytical solutions obtained by Giordano et al. [9].

For matrix-cracking, taking into account the microscopic elastic properties of the resin, the simulation shows that a separation of matrix cracking in "in-plane"-direction cannot be separated from matrix cracking in "out-of-plane"-direction. For unidirectional CFRP, the frequency distribution is determined by the elastic properties and not the direction of radiation, as expected from the theory of plate-wave excitation [16]. Taking into account the microscopic elastic properties of resin and fiber, the simulations show that a distinction of matrix-cracking and fiber-breakage can be made based on different intensity ratios of symmetric and antisymmetric plate-wave modes. A high intensity of antisymmetric modes corresponds to a high frequency contribution in the lower frequency range for matrix cracking, while high intensity of symmetric modes corresponds to a contribution in the higher frequency range for fiber breakage, as experimentally observed by various authors [1, 2, 3, 4, 5].

In addition to spectra dominated by antisymmetric or symmetric-modes, we find frequency spectra to which both modes contribute significantly. These are obtained by simultaneous excitation in $x$ - and z-direction. This excitation is expected in the case of fiber-matrix interface failure. The increase of the ratio of the displacement amplitudes $d_{x} / d_{z}$ results in a systematic shift of the frequency weight to higher frequencies.

A systematic variation of the z-position of the used model source results in significant changes of the frequency distribution, especially in the case of fiber breakage. Nevertheless, for all positions the frequency spectra obtained are dominated by the type of microscopic failure and can be classified accordingly by appropriate frequency based parameters. In particular, fiber-breakage and fiber-matrix interface failure can clearly be separated, as the latter one exhibits strong low frequency components that are not observed for fiber breakage.

The fact that the experimentally obtained frequency spectra can be described by the three failure mechanisms addressed by the simulation is confirmed by the results of the presented pattern recognition approach based on characteristic frequency parameters. This also yields three distinct signal types corresponding to matrix cracking, fiber breakage and fiber-matrix interface failure.

\section{Conclusions}

The similarity between the experimentally obtained and simulated frequency spectra in combination with the results of the presented pattern recognition methodology can act as proof of principle for the presented geometry, material and acoustic emission 
sensor type. For a generalized application of the presented approach to arbitrary CFRP-structures, influences on the detected frequency spectra arising from attenuation and geometry have to be further investigated. Especially the influence of dispersive wave propagation, the source position relative to the sensor and the stacking sequences of the CFRP can have a dominant influence on the obtained frequency spectra. For the presented CFRP specimen geometry we demonstrated that fiber breakage, matrix cracking and sources representing fiber-matrix interface failure result in characteristic frequency spectra of the simulated signals. These current simulations strongly support the suggestions of other authors to use frequency based criteria to distinguish between various failure mechanisms in CFRP under the condition, that attenuation arising from dispersion wave propagation is negligible $[1,17]$.

In particular, we suggest that an increase of $d_{x} / d_{z}$ describes an increase of adhesive strength between fiber and matrix, i. e. the acoustic emission analysis of the measured frequency spectra could yield a finger-print of the adhesive strength.

\section{Acknowledgments}

We thank A. Horoschenkoff from the University of Applied Science Munich for providing the specimens used in this investigation and U. Eckern, J. Moosburger-Will and $\mathrm{M}$. Klemm for the valuable discussions and suggestions.

\section{Literature}

[1] J. Bohse and J. Chen, "Acoustic emission examination of mode I, mode II and mixed-mode I/II interlaminar fracture of unidirectional fiber-reinforced polymers" J. Acoustic Emission 19 pp. 1-10 (2001)

[2] W. Haselbach, B. Lauke, "Acoustic emission of debonding between fibre and matrix to evaluate local adhesion" Composites Science and Technology 63 pp. 2155-2162 (2003)

[3] C. R. Ramirez-Jimenez, N. Papadakis, N. Reynolds, T.H. Gan, P. Purnell, M. Pharaoh, "Identification of failure modes in glass/polypropylene composites by means of the primary frequency content of the acoustic emission event", Composites Science and Technology 64 pp. 1819-1827 (2004)

[4] A. Marec, J.-H. Thomas, R. Guerjouma, „Damage characterization of polymer-based composite materials: Multivariable analysis and wavelet transform for clustering acoustic emission data“, Mechanical Systems and Signal Processing 22 pp. 1441-1464 (2008)

[5] X. Li, C. Ramirez, E. L. Hines, M. S. Leeson, P. Purnell, M. Pharaoh, „Pattern Recognition of Fiberreinforced Plastic Failure Mechanism using Computational Intelligence Techniques”, Neural Networks, IEEE World Congress on Computational Intelligence pp.2340-2345 (2008)

[6] M. Ohtsu, K. Ono, „A generalized theory of acoustic emission and Green's function in a half space“, J. Acoustic Emission 3 pp. 27-40 (1984)

[7] M. Ohtsu, K. Ono, "The generalized theory and source representation of acoustic emission", J. Acoustic Emission 5 pp.124-133 (1986)

[8] E. R. Green, “Acoustic Emission in Composite Laminates”, J. Nondestructive Evaluation 17:3, pp. 117-127 (1998)

[9] M. Giordano, L. Condelli, L. Nicolais, "Acoustic emission wave propagation in a viscoelastic plate", Composites Science and Technology 59 pp.1735-1743 (1999)

[10] E. R. Green, "Acoustic emission sources in a cross-ply laminated plate”, Composites Engin. 5 pp. 1453-1469 (1995)

[11] W. H. Prosser, M. A. Hamstad, J. Gary, A. O. Gallagher, „Finite Element and Plate Theory Modeling of Acoustic Emission Waveforms“, Journal of Nondestructive Evaluation 18:3 pp. 83-90 (1999) 
[12] M. Castaings, C. Bacon, B. Hosten, M. V. Predoi, "Finite element predictions for the dynamic response of thermo-viscoelastic material structures", Journal of the Acoustic Society of America, 115:3 pp. 1125-1133 (2004)

[13] J. H. Nieuwenhuis, J. Neumann, D. W. Greve, I. J. Oppenheim, „Generation and detection of guided waves using PZT wafer transducers“, IEEE Transactions Ultrasonics, Ferroelectrics and Frequency Control 52 pp. 2103-2111 (2005)

[14] D. W. Greve, J. J. Neumann, J. H. Nieuwenhuis, I. J. Oppenheim, N. L. Tyson, „Use of Lamb waves to monitor plates: experiments and simulations“, Proceedings of the Society of Photo-Optical Instrumentation Engineers, SPIE-5765 pp. 281-292 (2005)

[15] B. Hosten, "Heterogeneous structure of modes and Kramers-Kronig relationship inanisotropic viscoelastic materials", Journal of the Acoustic Society of America 104:3 pp. 1382-1388 (1998)

[16] M. A. Hamstad, A. O. Gallagher, J. Gary. "A Wavelet Transform Applied To Acoustic Emission Signals: Part 1: Source Identification”, Journal of Acoustic Emission, Vol. 20, pp. 39-61 (2002).

[17] W. H. Prosser, "Advanced AE Techniques in Composite Materials Research", Journal of Acoustic Emission, 14:3-4 pp. 1-11 (1996)

[18] L. B. Freund, "The initial wave front emitted by a suddenly extending crack in an elastic solid", Journal of Applied Mechanics 39 pp. 601-602 (1972)

[19] “COMSOL Multiphysics - Structural Mechanics Module", User's Guide, Comsol AB (2008)

[20] L. Goujon, J. C. Baboux, "Behaviour of acoustic emission sensors using broadband calibration techniques", Measurement Science and Technology 14 pp. 903-908 (2003)

[21] K. Ono, H. Cho, T. Matsuo, "Transfer functions of acoustic emission sensors", Journal of Acoustic Emission 26 pp. 72-90 (2008)

[22] J. Keprt, P. Benes, "The determination of uncertainty in the calibration of acoustic emission sensors", International Journal of Microstructure and Materials Properties 4:1 pp. 85-103 (2009)

[23] C. B. Scruby, H. N. G. Wadley, "A calibrated capacitance transducer for the detection of acoustic emission", J. Phys. D. Appl. Phys 11 pp. 1487-1494 (1978)

[24] H. Hatano, T. Chaya, S. Watanabe, K. Jinbo, "Reciprocity Calibration of Impulse Responses of Acoustic Emission Transducers", IEEE Transactions on Ultrasonics, Ferroelectrics and Frequency Control 45:5 pp. 1221-1228 (1998)

[25] M. G. R. Sause, D. Schultheiß, S. Horn, „Acoustic emission investigation of coating fracture and delamination in hybrid carbon fiber reinforced plastic structures", Journal of Acoustic Emission, Vol. 26, pp. 1-13 (2008).

[26] M. G. R. Sause, F. Haider, S. Horn, „Quantification of metallic coating failure on carbon fiber reinforced plastics using acoustic emission“, Surface and Coatings Technology 204:3 pp. 300-308 (2009)

[27] "NOESIS Advanced Acoustic Emission Data Analysis and Pattern Recognition \& Neural Networks Software for Acoustic Emission Applications", User's Manual, Enviroacoustics S.E. (2006).

[28] D. L. Davies, D. W. Bouldin, "A cluster separation measure”, IEEE T.

Pattern Anal. $1224-227$ (1979)

[29] J. T. Tou, "DYNOC - A dynamic optimal cluster-seeking technique", International Journal of Computer and Information Sciences, Vol. 8:6, pp. 541-547 (1979)

[30] A. A. Anastassopoulos and T. P. Philippidis, "Clustering Methodology for the Evaluation of Acoustic Emission from Composites", Journal of Acoustic Emission, Vol. 13, pp. 11-21 (1995).

[31] M. G. R. Sause, "AWARE++ Software manual Rev. 1.0",

http://www.physik.uni-augsburg.de/exp2/downloads.de.html, (2009)

[32] P.D. Wilcox, C.K. Lee, J.J. Scholey, M.I. Friswell, M.R. Wisnom, and B. W. Drinkwater, "Progress Towards a Forward Model of the Complete Acoustic Emission Process", Advanced Materials Research Vol. 13-14 pp. 69 - 75 (2006)

[33] J.J. Scholey, P.D. Wilcox, C.K. Lee, M.I. Friswell and M.R. Wisnom, "Acoustic Emission in Wide Composite Specimens", Advanced Materials Research Vol. 13-14 pp. 325 - 332 (2006)

[34] C.B. Scruby and D.J. Buttle, "Quantitative Fatigue Crack Measurement by Acoustic Emission." in Crack Measurement: Techniques and Applications (eds K J Marsh \& R Smith) EMAS, UK, p. 207. (1992)

[35] W.H. Prosser, K.E. Jackson, S. Kellas, B.T. Smith, J. McKeon and A. Friedman, "Advanced Waveform-Based Acoustic Emission Detection of Matrix Cracking in Composites", Materials Evaluation, Vol. 53:9, pp. 1052-1058 (1995), 